\title{
The formation of ultra compact dwarf galaxies and massive globular clusters
}

\section{Quasar-like objects to test for a variable stellar initial mass function}

\author{
T. Jeřábková ${ }^{1,2}$, P. Kroupa ${ }^{2,1}$, J. Dabringhausen ${ }^{1}$, M. Hilker $^{3}$, and K. Bekki ${ }^{4}$ \\ 1 Astronomical Institute, Charles University in Prague, V Holešovičkách 2, 18000 Praha 8, Czech Republic \\ e-mail: tjerabko@eso.org \\ 2 Helmholtz Institut für Strahlen und Kernphysik, Universität Bonn, Nussallee 14-16, 53115 Bonn, Germany \\ e-mail: pavel@astro.uni-bonn.de \\ 3 European Southern Observatory, Karl-Schwarzschild-Str. 2, 85748, Garching bei München, Germany \\ 4 ICRAR, The University of Western Australia, 35 Stirling Hwy, 6009 Crawley Western Australia, Australia
}

Received 24 May 2017 / Accepted 21 August 2017

\begin{abstract}
The stellar initial mass function (IMF) has been described as being invariant, bottom-heavy, or top-heavy in extremely dense starburst conditions. To provide usable observable diagnostics, we calculate redshift dependent spectral energy distributions of stellar populations in extreme star-burst clusters, which are likely to have been the precursors of present day massive globular clusters (GCs) and of ultra compact dwarf galaxies (UCDs). The retention fraction of stellar remnants is taken into account to assess the mass to light ratios of the ageing star-burst. Their redshift dependent photometric properties are calculated as predictions for James Webb Space Telescope (JWST) observations. While the present day GCs and UCDs are largely degenerate concerning bottom-heavy or top-heavy IMFs, a metallicity- and density-dependent top-heavy IMF implies the most massive UCDs, at ages $<100$ Myr, to appear as objects with quasar-like luminosities with a $0.1-10 \%$ variability on a monthly timescale due to core collapse supernovae.
\end{abstract}

Key words. galaxies: formation - galaxies: star clusters: general - galaxies: high-redshift - galaxies: dwarf - quasars: general stars: luminosity function, mass function

\section{Introduction}

The question of whether the stellar initial mass function (IMF) varies systematically with the physical conditions is a central problem of modern astrophysics (Elmegreen 2004; Bastian et al. 2010; Kroupa et al. 2013). If it does vary, we would expect the largest differences compared to nearby star forming regions in the most extreme star-burst conditions (e.g. Larson 1998). Where are the most extreme star-burst conditions in terms of star formation rate densities to be found, with the constraint that their present-day remnants are observable allowing detailed observational scrutiny of their present-day stellar populations? Given the present-day masses $\left(>10^{5} M_{\odot}\right)$ and present-day half-light radii $(1-50 \mathrm{pc})$, globular clusters (GCs) and in particular ultra compact dwarf galaxies (UCDs) are promising candidates (Mieske et al. 2002; Chilingarian et al. 2008; Brodie et al. 2011, present-day half mass radius). For example a $10^{7} M_{\odot}$ presentday UCD with a half-light radius of $20 \mathrm{pc}$ would likely have had a mass of $10^{8} M_{\odot}$ and a radius of a few pc when it was 1 Myr old (Dabringhausen et al. 2010).

Ultra compact dwarf galaxies were discovered by Hilker et al. (1999) and Drinkwater et al. (2000). There is no unique definition of what a UCD is, but it is generally taken to mean a stellar system with a radius between a few and $100 \mathrm{pc}$, and a dynamical mass of $10^{6} M_{\odot} \lesssim M_{\text {dyn }} \lesssim 10^{8} M_{\odot}$. Absolute magnitudes of UCDs lie roughly between $-10 \gtrsim M_{V} \gtrsim-16 M_{V}$, where the higher value corresponds to an old and low mass UCD (similar to $\omega$ Cen), while the lower value is more characteristic for a fairly young and/or massive UCDs (like W3). The most massive known old UCDs have magnitudes of $M_{V} \approx-14$. The presence of UCDs has been reported also in other galaxy clusters, for example Virgo (Drinkwater et al. 2004), Coma (Price et al. 2009), Centaurus (Mieske et al. 2007), Hydra I (Misgeld et al. 2011), and Perseus (Penny et al. 2012), or in massive intermediate-redshift clusters (e.g. Zhang \& Bell 2017). The existence of systems with properties of UCDs was discussed already by Kroupa (1998) based on the observations of star cluster complexes in the Antennae galaxies.

The origin and evolution of UCDs is still a matter of debate (e.g. Côté et al. 2006; Hilker 2009; Brodie et al. 2011) and up until today several possible formation scenarios have been proposed: (A) UCDs are the massive end of the distribution of GCs (e.g. Mieske et al. 2002; Forbes et al. 2008; Murray 2009; Dabringhausen et al. 2009; Chiboucas et al. 2011; Mieske et al. 2012; Renaud et al. 2015); (B) UCDs are merged star cluster complexes (Kroupa 1998; Fellhauer \& Kroupa 2002b,a; Brüns et al. 2011); (C) UCDs are the tidally stripped nuclei of dwarf galaxies (Oh et al. 1995; Bekki et al. 2001, 2003; Drinkwater et al. 2003; Goerdt et al. 2008; Pfeffer \& Baumgardt 2013); and (D) UCDs are remnants of primordial compact galaxies (Drinkwater et al. 2004).

Murray (2009) showed that the distribution and kinematics of the visible matter is not consistent with UCDs being 
objects dominated by non-baryonic dark matter halos. This is supported by a detailed study of one of the most massive UCDs (Frank et al. 2011). Based on the Millenium II cosmological simulation, Pfeffer et al. (2014) show that the formation of UCDs as tidally stripped nuclei of dwarf galaxies can account only for about 50 per cent of observed objects with mass $>10^{7} M_{\odot}$; for masses $>10^{6} M_{\odot}$ this drops to approximately 20 per cent (Pfeffer et al. 2016). Furthermore, earlier Thomas et al. (2008) showed that the tidally stripped nucleus scenario fails to reproduce UCDs located in the outer parts of the Fornax cluster. Based on these results we focus on scenarios (A) and (B), which both suggest that UCDs are the high-mass end of star cluster-like objects, formed most likely during massive starbursts (Weidner et al. 2004; Schulz et al. 2015, 2016) at higher redshift, where compact star-bursts are indeed observed (Vanzella et al. 2017; Glazebrook et al. 2017).

In this contribution we aim to quantify how extreme star formation environments may appear at high redshifts, where the most intense star-bursts are likely to have occurred. We therefore concentrate on the progenitors of present-day UCDs and GCs (i.e. their young counterparts at high redshift). We construct stellar population models using the PEGASE ${ }^{1}$ (Fioc \& Rocca-Volmerange 1997) code to suggest possible photometric diagnostics to provide observational predictions for upcoming missions such as the James Webb Space Telescope (JWST). The underlying question for this work is: Can a systematic variation of the stellar IMF be confirmed by observations of the likely high-redshift precursors of present-day GCs and UCDs using the JWST?

This paper is structured as follows: the first section is devoted to the introduction of the topic. Section 2 describes the methods we use. Section 3 focuses on results and Sects. 4 and 5 contain the discussion and conclusion, respectively.

\section{Methods}

We compute properties of UCDs and UCD progenitors assuming that they have formed according to scenario (A), that is by monolithic collapse. In such a case we expect that the first UCDs were formed alongside the formation of early massive galaxies $(\approx 0.5$ Gyr after the Big Bang). Results obtained according to scenario (A) can be used to discuss and constrain also scenario (B), which is the formation of UCDs through mergers of cluster complexes. Since here we assume that GCs are low mass UCDs, we from here on refer to GCs also as (low-mass) UCDs.

\subsection{Parametrization of the UCDs}

For quantifying our approach, we consider UCDs with the following properties: the UCD's initial stellar mass, $M_{\mathrm{UCD}} \in$ $\left[10^{6}, 10^{9}\right] M_{\odot}$, red-shift, $z \in 0,3,6,9$, corresponding, respectively, to ages from the Big-Bang $\approx 13.5,2.1,0.9,0.6 \mathrm{Gyr}$ as demonstrated in Fig. A.1. For the redshift computations we use the standard $\Lambda$ cold dark matter $(\Lambda \mathrm{CDM})$ cosmology with Planck estimates, $\Omega_{\mathrm{m}} \approx 0.308, \Omega_{\Lambda} \approx 0.692$, $H_{0} \approx 67.8 \mathrm{~km} \mathrm{~s}^{-1} \mathrm{Mpc}^{-1}$ (Planck Collaboration XIII 2016; Planck Collaboration Int. XLVII 2016). Other parameters are metallicity, $[\mathrm{Fe} / \mathrm{H}]=-2$ and 0 , and the stellar initial mass function (IMF).

\footnotetext{
1 WWW . iap.fr/pegase
}

\subsection{The stellar IMF}

We describe the stellar IMF as a multi-power law,

$\xi\left(m_{\star}\right)= \begin{cases}k_{1} m^{-\alpha_{1}} & 0.08 \leq m_{\star} / M_{\odot}<0.50, \\ k_{2} m^{-\alpha_{2}} & 0.50 \leq m_{\star} / M_{\odot}<1.0, \\ k_{2} m^{-\alpha_{3}} & 1.00 \leq m_{\star} / M_{\odot} \leq 120,\end{cases}$

where

$\xi\left(m_{\star}\right)=\mathrm{d} N / \mathrm{d} m_{\star}$

is the number of stars per unit of mass and $k_{i}$ are normalization constants which also ensure continuity of the IMF function. As a benchmark we use the IMF $\alpha$ values derived from the Galactic star forming regions by Kroupa (2001), where $\alpha_{1}=1.3$ and $\alpha_{2}=$ $\alpha_{3}=2.3$, here denoted as CAN IMF (canonical IMF).

A larger $\alpha_{1}$ than the canonical value leads to a bottom-heavy IMF. A bottom-heavy IMF is also described by a single Salpeter slope $\alpha=\alpha_{1}=\alpha_{2}=\alpha_{3}=2.3$, which has been shown to lead to slightly elevated $M / L_{V}$ values (Dabringhausen et al. 2008; Mieske \& Kroupa 2008) relative to the CAN IMF. Throughout this paper we will call this IMF the SAL IMF (Salpeter IMF). An even more bottom-heavy IMF, which might be necessary for explaining the observed $M / L_{V}$ values around ten and higher, was suggested by van Dokkum \& Conroy (2010) for massive elliptical galaxies, due to features observed in their spectra (but see Smith \& Lucey 2013). This IMF is here referred to as the vDC $\mathrm{IMF}$, and is characterized by an IMF slope $\alpha=\alpha_{1}=\alpha_{2}=\alpha_{3}=$ 3.0. A dependency of $\alpha$ on star-cluster-scale star-formation density and metallicity is currently not known. Chabrier et al. (2014) suggest increased turbulence to account for a bottom-heavy IMF in dense star forming regions. However, further theoretical work (Bertelli Motta et al. 2016; Liptai et al. 2017) casts doubts on this. This issue clearly needs further research.

The other option of how to explain the observed $M / L_{V}$ ratios is a top-heavy IMF (Dabringhausen et al. 2009). The top-heavy IMF has an empirical prescription, which establishes the slope of the heavy-mass end, $\alpha_{3}$, over the interval of masses $(1,120) M_{\odot}$ as a function of metallicity $[\mathrm{Fe} / \mathrm{H}]$ and birth density of the embedded star cluster, $\varrho_{\mathrm{cl}}$. The lower stellar masses $\left(<1.0 M_{\odot}\right)$ are in our formulation here distributed according to the CAN IMF. The relation for $\alpha_{3}$ by Marks et al. (2012) is,

$\alpha_{3}= \begin{cases}2.3 & \text { if } x<-0.87 \\ -0.41 x+1.94 & \text { if } x \geq-0.87\end{cases}$

where

$x=-0.14[\mathrm{Fe} / \mathrm{H}]+0.99 \log _{10}\left(\frac{\varrho_{\mathrm{cl}}}{10^{6} M_{\odot} \mathrm{pc}^{-3}}\right)$.

These relations have been obtained from a multi-dimensional regression of GC and UCD data. To calculate the birth density, $\varrho_{\text {cl }}$, we use the empirical relation from Marks \& Kroupa (2012), where the half-mass radii of embedded clusters follow

$R_{\mathrm{cl}} / \mathrm{pc}=0.1\left(M_{\mathrm{ecl}} / M_{\odot}\right)^{0.13}$

where $M_{\text {ecl }}$ is the stellar mass. The total density (gas + star), $\varrho_{\mathrm{cl}}$, for a star formation efficiency, $\epsilon$, is given by

$\varrho_{\mathrm{cl}}=\frac{3 M_{\mathrm{ecl}}}{4 \epsilon \pi R_{\mathrm{cl}}^{3}}$

Further on, we assume a star formation efficiency $\epsilon=0.33$ (e.g. Megeath et al. 2016; Banerjee 2017) and refer to the IMF derived using these relation as the MKDP IMF. The 
Table 1. Summary of IMF variations used in this paper.

\begin{tabular}{ccccc}
\hline \hline IMF & $\begin{array}{c}\text { Top-heavy } \\
\text { MKDP }\end{array}$ & $\begin{array}{c}\text { Canonical } \\
\text { CAN }\end{array}$ & $\begin{array}{c}\text { Bott.-heavy } \\
\text { SAL }\end{array}$ & $\begin{array}{c}\text { Bott.-heavy } \\
\text { vDC }\end{array}$ \\
\hline$\alpha_{1}$ & 1.3 & 1.3 & 2.3 & 3.0 \\
$\alpha_{2}$ & 2.3 & 2.3 & 2.3 & 3.0 \\
$\alpha_{3}$ & $0.6-2.3$ & 2.3 & 2.3 & 3.0 \\
\hline
\end{tabular}

Notes. The $\alpha$-coefficients are defined by Eq. (1). The MKDP IMF depends on the initial stellar mass of the system according to Eq. (3).

$\alpha_{3}$ and half-mass radii $R_{\mathrm{cl}}$ are listed in Table 2. Theoretical arguments for the IMF becoming top-heavy with increasing density, temperature, and decreasing metallicity of the starforming gas cloud have been described by e.g. Larson (1998), Adams \& Laughlin (1996), Dib et al. (2007), Papadopoulos (2010) and Romano et al. (2017).

All the IMFs considered here are summarized in Table 1.

\subsection{PEGASE parameters}

The above parametrizations are used as input values to the PÉGASE time-dependent stellar population synthesis code ${ }^{2}$ (Fioc \& Rocca-Volmerange 1997) and we compute the time evolution of various quantities, such as the total luminosity, $L_{\mathrm{UCD}}$, number of massive stars $\left(m_{\star}>8 M_{\odot}\right), N_{\text {mass }}$, and also observable properties: the redshifted time evolution of the spectral energy distribution (SED), and colour-magnitude diagrams. Furthermore we investigate in detail the $M / L_{V}$ values. Besides the values considered here for the initial stellar mass, metallicity, and redshift of the UCDs $\left(M_{\mathrm{UCD}},[\mathrm{Fe} / \mathrm{H}], z\right)$, we use the following values to compute the PÉGASE models: a conservative value $\eta=0.05$ for the fraction of binaries producing supernovae Ia (e.g. Maoz 2008, where $\eta \in(0.05,0.4))$, zero inclination, no galactic winds, no in-fall matter, and no metallicity evolution (this would require a more complicated time-dependent IMF prescription and a star formation history). We take into account nebular emission and we assume no extinction since it can be corrected based on various assumptions in the further interpretation of our models. It is, however, worth mentioning that young and intermediate-age massive clusters in the local universe seem to be gas- and dust-free very early on (e.g. Bastian \& Strader 2014; Longmore 2015). The star formation history is assumed to have the shape of a step function with a non zero value for the first $5 \mathrm{Myr}$. We will compare this with the instantaneous starburst case, which has the same parameters apart from the fact that all stars form at the same time.

\subsection{Calculating $M / L_{V}$ ratios}

To evaluate the dynamical mass, $M$, and luminosity in the $V$ band, $L_{V}$, several assumptions are made: (i) we assume that the UCDs are gas free during the whole period of their evolution, which means that our predictions are only valid for pure stellar populations. (ii) Mass loss from the UCDs is only through stellar evolution in the form of ejected gas. We assume that all stars are kept in the system and no stars are lost by dynamical evolution of the UCDs. Stellar loss due to dynamical evolution is an important process (e.g. Balbinot \& Gieles 2017)

\footnotetext{
2 wWW.iap.fr/pegase
}

which is, however, significant only for systems with initial stellar mass $\lesssim 10^{6} M_{\odot}$ (Lamers et al. 2005; Schulz et al. 2016; Brinkmann et al. 2017). The reason is that for large stellar mass, $\gtrsim 10^{6} M_{\odot}$, the tidal radius in a Milky-Way galaxy potential is $\gtrsim 100$ pc for Galactocentric distances $\gtrsim$ few kpc such that the vast majority of stars remain bound. This is consistent with using Eq. (5) from Lamers et al. (2005) which, after integration, gives an upper limit for tidal stellar mass loss over a Hubble time $\approx 10^{3} M_{\odot}$ independent of initial mass. (iii) The effect of loss of dark remnants (neutron stars and black holes) on the estimate of the dynamical mass for the vDC, SAL, and CAN IMF is negligible to the value of the $M / L_{V}$ ratio (for the CAN IMF dark remnants contribute only few per cent of the UCD mass). However, in the MKDP IMF case, where dark remnants contribute a substantial fraction to the total mass of the system, the amount of dark remnants kept is no longer negligible. The actual fraction of dark remnants kept in a system is still being discussed mainly because to study remnant ejections, close dynamical encounters in a system are important on a Hubble timescale (Banerjee 2017). Such studies are extremely computationally intensive for systems as massive as UCDs. On the other hand, Peuten et al. (2016) and Baumgardt \& Sollima (2017), constrain the retention fraction of dark remnants in lower mass GCs, which allows us to deduce implications for UCDs.

\subsubsection{Retention fraction of dark remnants}

The mass-to-light ratios of UCDs depend on the retention fraction of stellar remnants within the system once the progenitor stars die. In order to assess the possible range of retention fractions, we assume that white dwarfs (WDs) receive no kicks upon the death of their progenitor stars such that all WDs remain bound to the system. A star more massive than $8 M_{\odot}$ explodes as a type II supernova or implodes leaving either a neutron star or a stellar black hole. The kicks these receive during such violent events due to the asymmetry of the explosion or implosion are uncertain. Large natal kicks will lead to the loss of most such remnants from the system.

We estimate the retention fraction by assuming that 10 per cent of all neutron stars and black holes are retained in a globular cluster with a mass of $10^{5} M_{\odot}$. This is a conservative assumption as Baumgardt \& Sollima (2017) and Peuten et al. (2016) constrain the retention fraction to be less than about 50 per cent for globular clusters (GCs) based on a detailed study of their observed mass segregation. With this normalisation condition, and assuming two possible radius-mass relations for GCs and for UCDs, we can estimate the likely values of the retention fraction of stellar remnants (neutron stars and black holes) as a function of birth system mass, $M$. One possibility for the $R(M)$ relation is to assume the observed radii of clusters (typically about $3 \mathrm{pc}$ ) and of UCDs (Eq. (4) in Dabringhausen et al. 2008). The other possibility is to assume that the stars die in their birth systems before these expand (Dabringhausen et al. 2010) due to residual gas expulsion and stellar-evolution-driven mass loss with the $R(M)$ relation constrained by Marks \& Kroupa (2012, their Eq. (7)). Given the $R(M)$ relation, we assume that remnants are lost if their speed after the kick is larger than the central escape velocity. We assume the systems to be Plummer models and that the kick velocities follow a Maxwell-Boltzmann distribution (MBD) with velocity dispersion $\sigma_{\text {kick }}$.

Figure 1 shows the resulting MBD for the two assumed $R(M)$ relations. The adopted normalization condition forces the MBD to be narrow, with a small kick, $\sigma_{\text {kick }}=24 \mathrm{~km} \mathrm{~s}^{-1}$, for the 
Table 2. Values of the initial radii of the embedded star clusters, $R_{\mathrm{cl}}$, (Marks \& Kroupa 2012), $\alpha_{3}$ values (see Eq. (3)), and the number of massive stars $N\left(m_{\star}>8 M_{\odot}\right)$ computed as a function of initial stellar mass, $M_{\mathrm{UCD}}=M_{\mathrm{ecl}}$, and metallicity.

\begin{tabular}{cc|cccc|cc}
\hline \hline & & \multicolumn{3}{|c}{ MKDP IMF } & \multicolumn{2}{c}{ CAN IMF } \\
& & \multicolumn{2}{c|}{$[\mathrm{Fe} / \mathrm{H}]=-2$} & & \\
$M_{\mathrm{UCD}}\left[M_{\odot}\right]$ & $R_{\mathrm{cl}}[\mathrm{Fc}]$ & $\alpha_{3}$ & $10^{5} N\left(m_{\star}>8 M_{\odot}\right)$ & $\alpha_{3}$ & $10^{5} N\left(m_{\star}>8 M_{\odot}\right)$ & $\alpha_{3}$ & $10^{5} N\left(m_{\star}>8 M_{\odot}\right)$ \\
\hline $10^{6}$ & 0.6 & 1.61 & 0.2 & 1.73 & 0.2 & 2.3 & 0.1 \\
$10^{7}$ & 0.8 & 1.37 & 2.4 & 1.48 & 2.4 & 2.3 & 1.1 \\
$10^{8}$ & 1.1 & 1.12 & 23.2 & 1.23 & 23.8 & 2.3 & 10.9 \\
$10^{9}$ & 1.5 & 0.87 & 213.8 & 0.99 & 222.7 & 2.3 & 109.1 \\
$10^{10}$ & 2.0 & 0.62 & 1943.1 & 0.74 & 2032.6 & 2.3 & 1091.2 \\
\hline
\end{tabular}

Notes. For comparison, we also show $N\left(m_{\star}>8 M_{\odot}\right)$ for the CAN IMF. The predicted values of the embedded cluster/UCD initial radii, $R_{\mathrm{cl}}$, are small. However, due to stellar and dynamical evolution, UCDs expand by approximately a factor of ten (Dabringhausen et al. 2010). We use the $R_{\mathrm{cl}}$ values as an empirical extrapolation from GCs and therefore at UCD scales departures are possible.

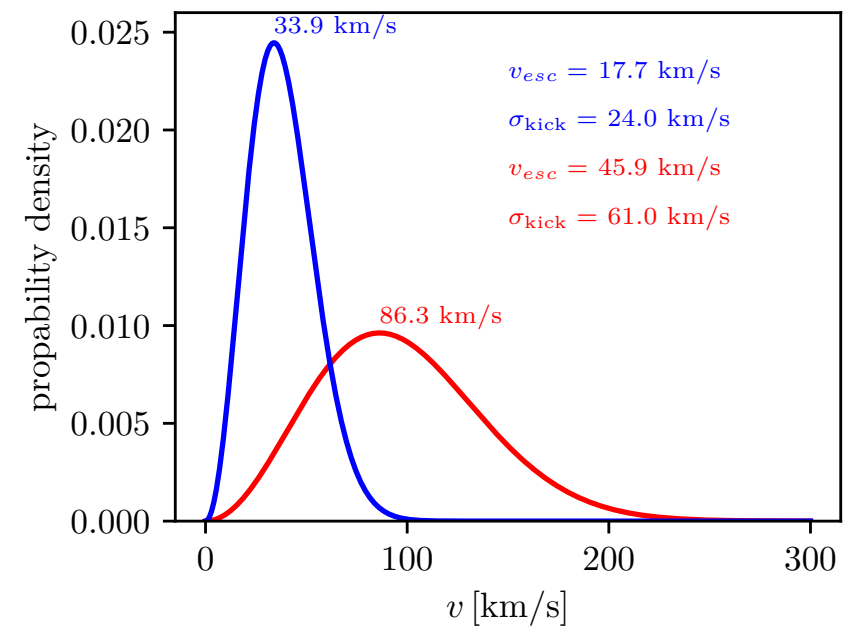

Fig. 1. Distribution function of speeds of dark stellar remnants. We use the conservative estimate that only 10 per cent of dark remnants are kept in $10^{5} M_{\odot}$ system due to SN kicks. We constrain the SN kick velocity distribution assuming it has a Maxwell-Boltzman shape for birth radius (Marks \& Kroupa 2012, red curve) and for present day radius, $3 \mathrm{pc}$ for GCs and for the UCDs we use the mass-radius relation from Dabringhausen et al. (2008; their Eq. (4), blue curve). The peak velocity, $\sigma_{\text {kick }}$, and $v_{\text {esc }}$ values are stated.

present-day radii of clusters and UCDs, while applying the birth radii results in a larger kick, $\sigma_{\text {kick }}=61 \mathrm{~km} \mathrm{~s}^{-1}$. Despite the two different values, the resulting retention fractions, defined for the present purpose as the fraction of remnants with a speed smaller than the central escape speed, are very similar. This is shown in Fig. 2, from which it also follows that the retention fraction increases steeply with system mass such that for $M>10^{6} M_{\odot}$ the retention fraction can be assumed to be near to 100 per cent, if the normalization condition applied here holds. We also plot the retention fraction for distributions with larger $\sigma_{\text {kick }}$ values, namely 100,300 , and $500 \mathrm{~km} \mathrm{~s}^{-1}$. Given these results it is reasonable to assume that the retention fraction of UCDs with birth masses larger than $10^{7} M_{\odot}$ is close to $100 \%$. Indirect evidence suggesting a possible large retention fraction in UCDs are recent findings of super massive black holes (SMBHs) in UCDs (e.g. Seth et al. 2014; Ahn et al. 2017, see Sect. 4.3). Nevertheless, we still investigate the effect of smaller retention fractions on the $M / L_{V}$ values to allow us to see the maximum impact the assumed IMF can potentially have.

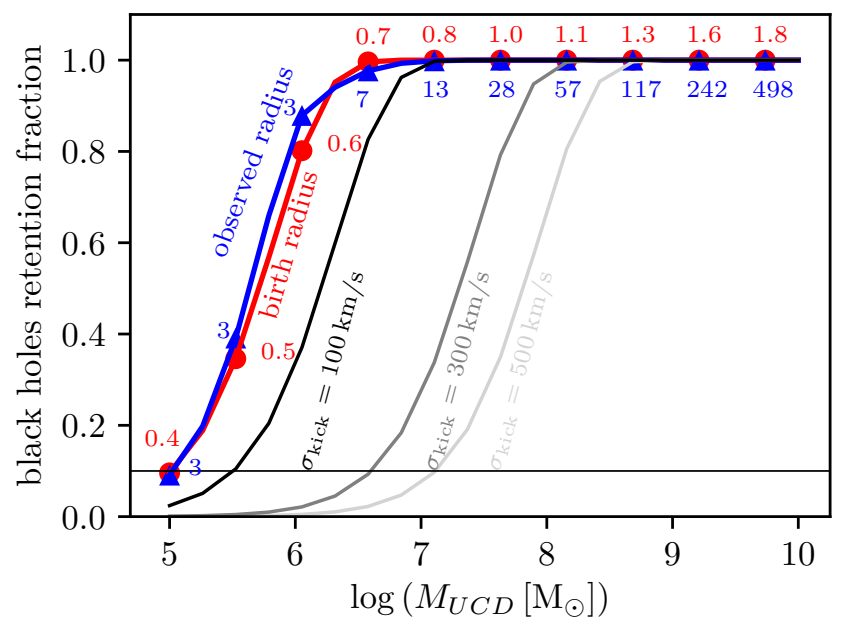

Fig. 2. Retention fraction of dark remnants as a function of system mass if we assume that the main mechanism of dark remnants ejection are SN kicks with a Maxwell-Boltzman kick velocity distribution shown in Fig. 1. We assumed two different mass-radius relations (red and blue curves, as in Fig. 1), radii for each mass being written next to the corresponding points and a normalization described in the text. In black and grey we plot retention fractions if larger kick velocity dispersions are assumed. In this case we use the mass-radius relation from Marks \& Kroupa (2012).

\subsection{Limitations of the models}

We note here three possible limitations to the models introduced above:

1. Effects of binaries. The majority of stars form in binaries (e.g. Marks et al. 2011; Thies et al. 2015). Mass transfer and mergers can rejuvenate stellar populations leading to significantly more UV radiation even after a few Gyr (Stanway et al. 2016, BPASS code). Since here we focus on the IR region mostly and on systems younger than $100 \mathrm{Myr}$, it is likely that our conclusions are not affected strongly if binary-star evolution is taken into account, but it is nevertheless important to check this factor in the future.

2. Multiple populations. It is well established that GCs have multiple stellar populations (e.g. Renzini et al. 2015, review). This may be true for UCDs as well. Future SED modelling has the potential to address how and if these can be observed in young systems at high redshift. However, we caution that binary stellar evolution (Item 1 above) may lead to degeneracies. 


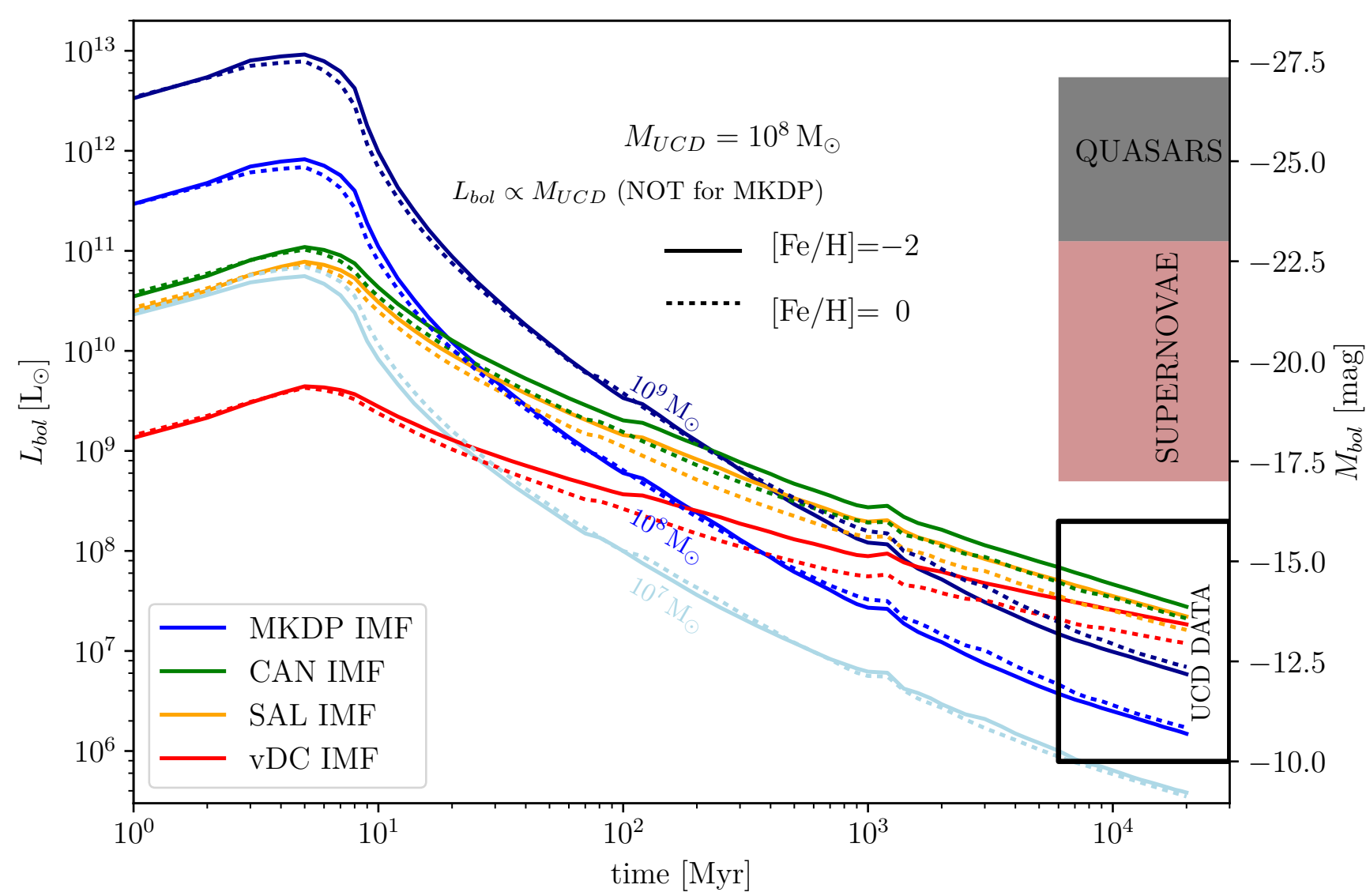

Fig. 3. Time evolution of the bolometric luminosity for different IMFs. The MKDP IMF changes with the initial mass of the UCD and does not scale linearly with its mass. In contrast, the vDC, SAL, and CAN IMFs have UCD-mass-independent slopes and scale proportionally with $M_{\mathrm{UCD}}$. The grey panel shows the typical luminosities of quasars (Dunlop et al. 1993, 2003; Souchay et al. 2015) and the brown panel shows the luminosity span for the peak luminosities supernovae (Gal-Yam 2012; Lyman et al. 2016), which might cause luminosity variations in $L_{\mathrm{bol}}$ of UCDs younger than about $50 \mathrm{Myr}$ according to SNe rates.

3. Statistical importance. Large statistical samples may be needed to ascertain a systematic variation of the IMF with physical conditions (Dabringhausen et al. 2008, 2009, 2012).

\section{Results}

For the grid of chosen parameters we construct a time grid (1-10 Myr with 1 Myr step, 10-100 Myr with 10 Myr steps, 100-1000 Myr with 100 Myr steps, and 1-13 Gyr with 1 Gyr steps) of SEDs, which contain all the light information from the source necessary for the construction of other observables. This SED data set will be published together with this paper. Further details (e.g. comparison of the PEGASE code and the StarBurst99-code (Leitherer et al. 1999) with description of the evolution of SEDs with time and dependency on redshift) can be found in Appendix A.

\subsection{Evolution of the bolometric luminosity with time}

To demonstrate how luminous progenitors of UCDs could have been, we compute the bolometric luminosity, $L_{\mathrm{bol}}$, as a function of time. The $L_{\mathrm{bol}}(t)$ dependency is shown in Fig. 3. The benchmark initial mass is $M_{\mathrm{UCD}}=10^{8} M_{\odot}$. For the cases of the CAN, SAL, and vDC IMF, the $L_{\text {bol }}$ values are proportional to $M_{\mathrm{UCD}}$. The MKDP IMF is a function of $M_{\mathrm{UCD}}$ and therefore we plot $L_{\mathrm{bol}}(t)$ also for $M_{\mathrm{UCD}}=10^{7}$ and $10^{9} M_{\odot}$. The main difference
Table 3. Quasar (QSO) data.

\begin{tabular}{ccccc}
\hline \hline QSO & $z$ & $M_{V}$ & $M_{I}$ & $\mathrm{RQ} / \mathrm{RL}$ \\
\hline $0923+201$ & 0.193 & -24.6 & -24.28 & $\mathrm{RQ}$ \\
$2344+184$ & 0.138 & -23.6 & -23.80 & $\mathrm{RQ}$ \\
$1635+119$ & 0.147 & -23.1 & -22.39 & $\mathrm{RQ}$ \\
$1012+008$ & 0.185 & -24.3 & -25.39 & $\mathrm{RQ}$ \\
$1004+130$ & 0.241 & -25.7 & -24.90 & $\mathrm{RL}$ \\
$1020-103$ & 0.197 & -24.2 & -24.35 & $\mathrm{RL}$ \\
$2355-082$ & 0.211 & -23.0 & -23.71 & $\mathrm{RL}$ \\
\hline
\end{tabular}

Notes. The first column is the QSO identification, $z$ is the redshift, $M_{V}$ and $M_{I}$ are absolute magnitudes, and RQ/RL labels the QSO as being radio quiet (RQ) or radio loud (RL). We have not found any publication or catalogue presenting both the absolute magnitudes in the $V$ filter, $M_{V}$, and $I$ filter, $M_{I}$. Therefore, the data in the table use the catalogue by Souchay et al. (2015) containing the $M_{I}$ values. The $M_{V}$ values are taken from Dunlop et al. $(1993,2003)$.

between different MKDP IMFs (for different $M_{\mathrm{UCD}}$ ) is the slope of the luminosity as a function of time. For a more top-heavy IMF, the decrease of the luminosity with time is steeper.

There are several noticeable features in this figure: (i) for the MKDP IMF, UCDs with initial stellar mass $M_{\mathrm{UCD}} \gtrsim 10^{8} M_{\odot}$ are as bright as quasars (Dunlop et al. 1993, 2003; Souchay et al. 2015) for the first few $10^{7}$ yr. We use low-redshift quasars because for these we were able to find bolometric luminosities, but also absolute magnitudes in the $V$ and $I$ band. We note, 

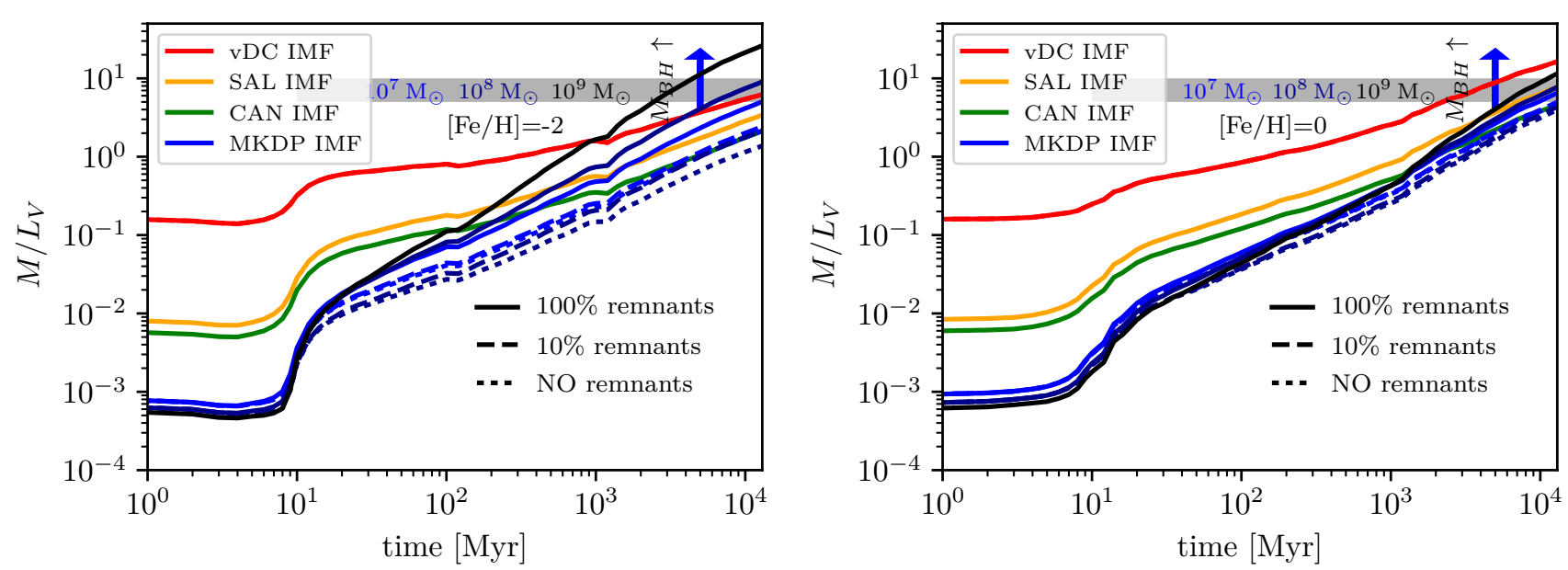

Fig. 4. Time evolution of $M / L_{V}$ for all IMFs considered (vDC, SAL, CAN, MKDP). We assume that the system is gas free and that neither stars nor remnants are leaving (the dashed lines show the change of the $M / L_{V}$ value if only certain fractions of remnants are kept). The upwards-pointing blue arrow demonstrates that if we would assume more massive remnants (e.g. due to implosion directly to a BH without a SN explosion), then this would lead to larger values of $M / L_{V}$. The models are computed for the case of $M_{\mathrm{UCD}}=10^{9} M_{\odot}$, however, results are mass independent. The only exception is the MKDP IMF, which depends on initial object mass, $M_{\mathrm{UCD}}$, and also contains a large fraction of mass in high mass stars, and is plotted for $M_{\mathrm{UCD}}=\left(10^{9}, 10^{8}, 10^{7} M_{\odot}\right.$, bottom to top) and different fractions of remnants retained $(100 \%, 10 \%, 0 \%)$. Left panel: the evolution for $[\mathrm{Fe} / \mathrm{H}]=-2$. Right panel: the evolution for $[\mathrm{Fe} / \mathrm{H}]=0$. The grey band indicates the span of the observed present-day $M / L_{V}$ values, approximately five to ten for the majority of UCDs (Mieske et al. 2008; Dabringhausen et al. 2009). The scales are identical for both panels.

however, that high redshift quasars show very comparable luminosities as shown for example by Mortlock et al. (2011). This is mainly due to the presence of a large number of $\mathrm{O}$ and $\mathrm{B}$ stars $\left(10^{6}-10^{7}\right)$ in the system. Due to stellar evolution and corecollapse supernova explosions, these UCDs will be variable on a timescale of months. As already suggested by Terlevich \& Boyle (1993), such objects might be confused with quasars, especially for example in large photometric surveys. (ii) After less than a 100 Myr, a strong degeneracy between the IMF and $M_{\mathrm{UCD}}$ appears. That is, for different $M_{\mathrm{UCD}}$ and different IMFs, similar luminosities comparable to those of observed UCDs occur. (iii) The metallicity is a second-order effect.

\subsection{Time evolution of the $M / L_{v}$ ratio}

The PEGASE output allows us to evaluate the mass to light $\left(M / L_{V}\right)$ ratios in arbitrary photometric filters since with the time evolution the code keeps information about the current stellar mass, mass in black holes and neutron stars, and also about the gas (non-consumed initial gas and the gas ejected by stars). Since the vDC or SAL (bottom-heavy) IMFs do not depend on the initial UCD mass, neither does the $M / L_{V}$ ratio. The situation is different for the MKDP IMF and we therefore plot the time evolution of the $M / L_{V}$ ratio for different initial masses in Fig. 4.

\subsection{1. $M / L_{v}$ ratio variations}

The results show that the largest differences in $M / L_{V}$ ratios are evident in the first $\approx 100 \mathrm{Myr}$, which is the age corresponding to the time when the most massive stars evolve into dark remnants (see Fig. 4 where we can also see the effect of the retention fraction of remnants on the $M / L_{V}$ values). For later times (>100 Myr), degeneracy among the data appears: the UCDs formed with a MKDP IMF are equally or less bright than the UCDs with a CAN or SAL IMF formed with the same initial mass. Even the $M / L_{V}$ ratios might become indistinguishable at times older than $\approx 100 \mathrm{Myr}$ for different IMFs within the observational and metallicity uncertainties.
Using the same models, we construct the dependence of $M / L_{V}$ on $L_{V}$ for different evolutionary times (Fig. 5). To construct these plots, we assumed the set of initial UCD masses to be $10^{7}, 10^{8}, 10^{9} M_{\odot}$. As is clear from the comparison of the panels in Fig. 5, the metallicity has a large effect on the $M / L_{V}$ values.

\subsection{The supernova rates}

The supernovae (SNe) II rate (Lonsdale et al. 2006; Anderson et al. 2011) can be a very good indicator of the IMF as one can see in Fig. 6. According to the standard stellar evolutionary tracks employed here, every star more massive than $8 M_{\odot}$ ends as a supernova explosion. However, this may not always be the case. As the metallicity varies it may happen that a star of a given mass may implode and create a black hole directly without any explosion (see e.g. Pejcha \& Thompson 2015) and therefore our theoretical prediction represents the upper limit to the SN II rate.

It is important to point out that the $\mathrm{SN}$ rate depends on the star formation history of a system. If the whole system is formed during an instantaneous starburst, the peak SN II rate might by a factor of ten higher than in the case of constant star formation over a period of $5 \mathrm{Myr}$. On the other hand, if the star formation is more extended then the period of high SN II rate lasts longer.

Considering the luminosities of SNe (Gal-Yam 2012; Lyman et al. 2016), in Fig. 3 we show that at the later phases, $>10 \mathrm{Myr}$, the SNe can reach up to 10 per cent of the UCD's total flux for the MKDP IMF and $10^{9} M_{\odot}$ initial mass (for smaller initial masses, SN II explosions will be more pronounced) and therefore might be detectable as photometric fluctuations on the scale of months.

To compute the SN Ia, rate we adopt a conservative fraction, $\eta=0.05$, of intermediate-mass stars that eventually explode as SNe Ia. According to $\mathrm{Maoz}$ (2008), $\eta=0.02-0.4$. We use $\eta=0.05$ also because it is a default value of this parameter and therefore our results will probably be comparable with other studies. The $\eta$ value affects the estimates of the number of SNe Ia and also of the ejecta (Thielemann et al. 1986; Greggio \& Renzini 1983; Matteucci \& Greggio 1986). 

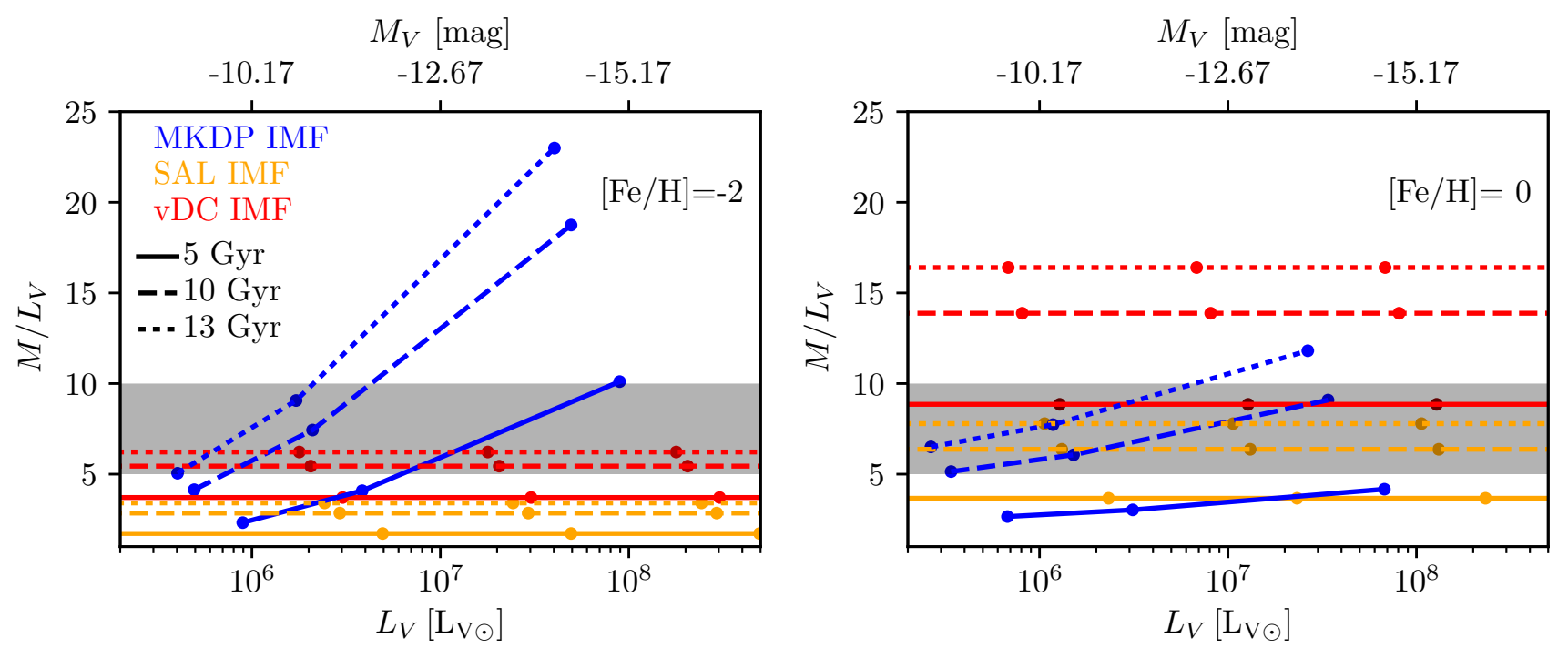

Fig. 5. Values of the $M / L_{V}$ ratio as a function of $L_{V}$ for three different times 5, 10, $13 \mathrm{Gyr}$. The case of the MKDP IMF is not constant since the MKDP IMF is a function of initial mass. The plotted curves assume all remnants are kept; if only a fraction are kept, the $M / L_{V}$ values would become smaller accordingly. There are two horizontal axes, the one on the top of the plots shows corresponding values of the absolute magnitude in the rest-frame $V$ band. The observed values of $M / L_{V}$ for UCDs are in the interval from approximately five to ten, with few values spanning up to 15 (Mieske et al. 2002), which are shown by the grey band. The points plotted on the curves mark luminosities and $M / L_{V}$ values for UCDs starting with initial mass of $10^{9}, 10^{8}$, and $10^{7} M_{\odot}$. Left panel: $[\mathrm{Fe} / \mathrm{H}]=-2$. Right panel: $[\mathrm{Fe} / \mathrm{H}]=0$.

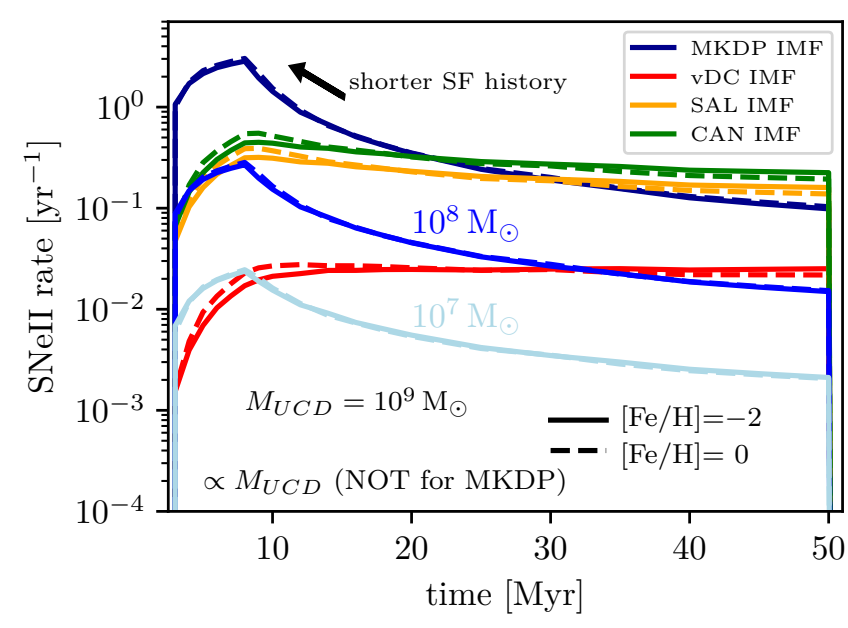

Fig. 6. SNe II rate as a function of time for a constant star formation history over a period of $5 \mathrm{Myr}$. In the instantaneous star-burst case, the SNeII rate may be $10 \times$ higher at ages $<10$ Myr. We show the case of the vDC, SAL, CAN, and MKDP IMFs. Since only the MKDP IMF does not scale linearly with initial stellar mass, $M_{\mathrm{UCD}}$, we show also the lines for $M_{\mathrm{UCD}}=10^{7} M_{\odot}$ and $10^{8} M_{\odot}$; for the other IMFs we chose to plot only $10^{9} M_{\odot}$. The arrow indicates that the peak of the SN II rate shifts to the left and upwards for a star formation history which is shorter than $5 \mathrm{Myr}$.

The computed rates are plotted in Fig. 7. Using a value as large as $\eta=0.4$ (Maoz 2008, upper value) would imply an eight times larger SN Ia rate. The other results, such as the luminosities or $M / L_{V}$ values, are not affected by the value of $\eta$, since different values of $\eta$ do not change the mass distribution of stars.

\subsection{Time evolution of the $\beta_{U V}$ slope}

The $\beta_{\mathrm{UV}}$ slope is defined here as the slope of the fitted linear function to the logarithmically scaled SED, expressed in units of $\operatorname{erg}^{-1} \mathrm{~cm}^{-2} \AA^{-1}$, in a wavelength interval $(1350,3500) \AA$ in the

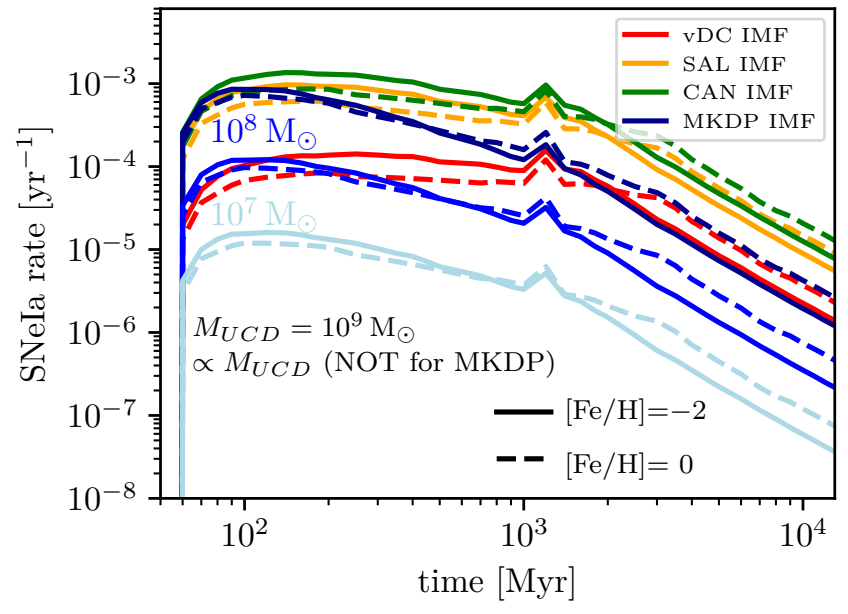

Fig. 7. SNe Ia rate as a function of time. We show the case of the vDC, SAL, CAN, and MKDP IMFs. Since only the MKDP IMF does not scale linearly with initial stellar mass, $M_{\mathrm{UCD}}$, we show also the lines for $M_{\mathrm{UCD}}=10^{7} M_{\odot}$ and $10^{8} M_{\odot}$; for the other IMFs we chose to plot only $10^{9} M_{\odot}$. The text gives more details.

rest frame of the observed object, as shown in Fig. A.7 in the Appendix. We computed $\beta_{\mathrm{UV}}$ for the complete set of our SEDs (Fig. 8). The $\beta_{\mathrm{UV}}$ values have been determined for objects down to $10^{6} M_{\odot}$ (for a top-heavy IMF this may be $10^{5} M_{\odot}$ ) at high redshifts (up to $z=6$ ) (Vanzella et al. 2017) and therefore might allow very useful additional constraints on the IMF and the age estimates. The $\beta_{\mathrm{UV}}$ values are metallicity sensitive and have a generally increasing trend with age. The dependence on the IMF is stronger for objects younger than $10 \mathrm{Myr}$ and at low metallicity, $\beta_{\mathrm{UV}}^{\mathrm{MKDP}} \approx-2.1, \beta_{\mathrm{UV}}^{\mathrm{vDC}} \approx-2.6$ at age $1 \mathrm{Myr}$ for $[\mathrm{Fe} / \mathrm{H}]=$ -2 . At an age of $200 \mathrm{Myr}$, these values evolve to $\beta_{\mathrm{UV}}^{M K D P} \approx-1.3$, $\beta_{\mathrm{UV}}^{\mathrm{vDC}} \approx-1.1$.

At a high redshift it may not always be possible to obtain a spectrum of a UCD. In such a case we can obtain photometric 

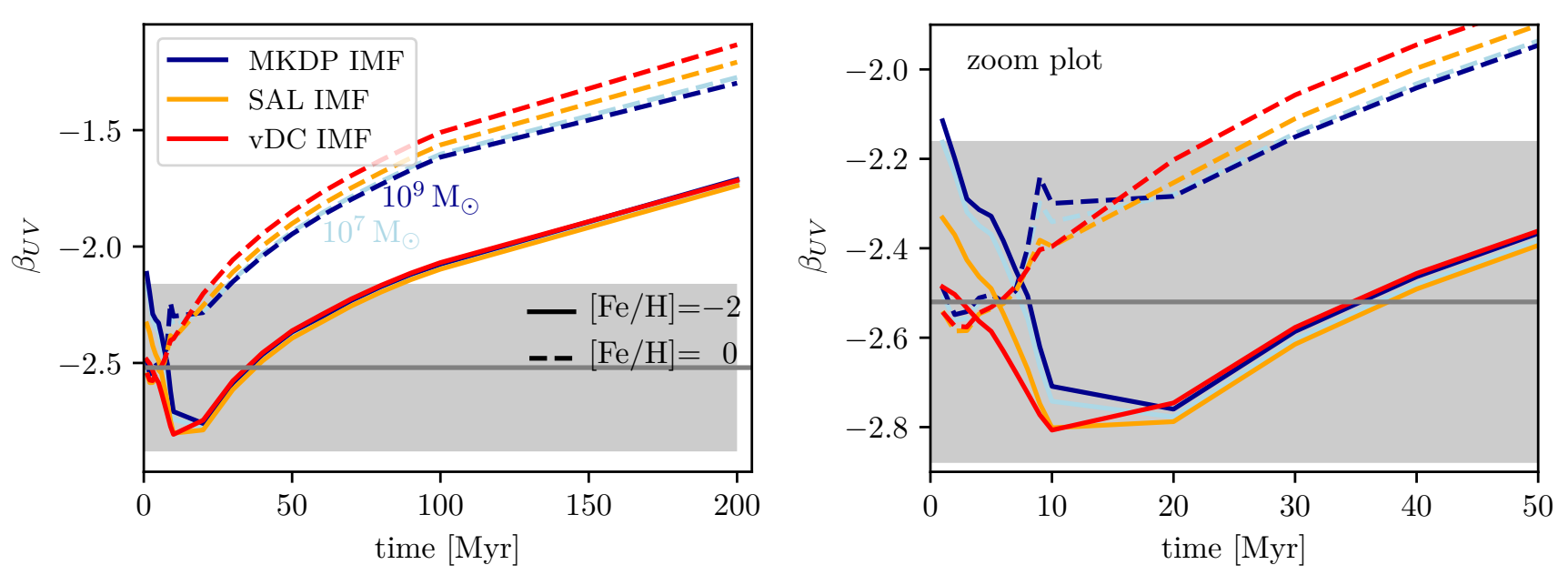

Fig. 8. Time evolution of the $\beta_{\mathrm{UV}}$ values fitted over the wavelength interval $(1350,3500) \AA$ to the SEDs in the rest frame of the observed object in units $\mathrm{erg}^{-1} \mathrm{~cm}^{-2} \AA^{-1}$. The $\beta_{\mathrm{UV}}$ slope is $M_{\mathrm{UCD}}$-independent, but not for the case of the MKDP IMF for which we plot the case of $M_{\mathrm{UCD}}=10^{7} M_{\odot}$ and $10^{9} M_{\odot}$. Left panel: the time evolution of the $\beta_{\mathrm{UV}}$ slope over the time period of $200 \mathrm{Myr}$. Right panel: the zoom-in plot covers the first $50 \mathrm{Myr}$. The grey band shows the measurement from Vanzella et al. (2017) for their object GC1.

fluxes in at least two filters and use these to approximate the $\beta_{\mathrm{UV}}$ slope. For $z=3$ one could, for example, use the JWST NIRCam instrument with filters $F 070 W$ and $F 115 W$. For $z=6$ and $z=9$ it is possible to use the same instrument but with filters $F 090 \mathrm{~W}$, $F 200 W$, and $F 115 W, F 300 M$, respectively.

\subsection{The colour-magnitude and the colour-colour diagrams}

Other observables, which can be computed from the SED and for standard filters and which are provided by PEGASE, are various colours and magnitudes. The time evolution of our objects is shown in the $V$ vs. $V-I_{\text {c }}$ diagram for comparison with other work (e.g. Evstigneeva et al. 2008). This is done for the CAN, SAL, and vDC IMFs in Fig. 10, and for the MKDP IMF in Fig. 9. As expected we can see the increasingly strong degeneracy with time that makes it hard to distinguish the metallicity, initial mass, or the IMF.

The colour-colour diagram has the advantage of having differences in magnitudes on both axis and therefore in a way subdues the information about the absolute values and enhances the differences in spectral shapes and features. As is shown in Fig. A.8, we chose to use the standard filters $J, K$, and $N$. The $N$ filter is almost identical to $F 1000 W$ on the mid-infrared instrument (MIRI) on the JWST. The $J$ and $K$ filters cover a similar range as the $F 115 \mathrm{~W}$ and $F 200 \mathrm{~W}$ filters on near infrared camera (NIRCam) on the JWST. The results presented here assume transmission functions corresponding to their filters to be given by the rectangular regions shown in Fig. A.4. These filters possess several useful characteristics: (i) even for a redshift of value nine, they are still in the spectral range covered by the PEGASE SEDs and therefore we do not introduce any additional errors by using the extrapolated range of the SEDs; (ii) $J, K$, and $N$ cover large parts of the SEDs and therefore are good representatives of an overall shape; and (iii) the $N$ filter is still in the well-described region where we do not expect large discrepancies from model to model and therefore also in real measurements as can be seen in Fig. A.2.

\subsection{Predictions for JWST}

The first question which we need to ask is whether UCD progenitors are bright enough to be detected and if so up to which
Table 4. Predictions for different filters of the JWST telescope.

\begin{tabular}{ccccc}
\hline \hline Instr. & Filter & $z$ & $S / N$ CAN & $S / N$ MKDP \\
\hline NIRCam & $F 115 W$ & 3 & 47 & 194 \\
NIRCam & $F 200 W$ & 3 & 47 & 215 \\
NIRCam & $F 480 M$ & 3 & 7 & 52 \\
MIRI & $F 1000 W$ & 3 & 0.4 & 4 \\
\hline NIRCam & $F 115 W$ & 6 & 14 & 76 \\
NIRCam & $F 200 W$ & 6 & 15 & 83 \\
NIRCam & $F 480 M$ & 6 & 2 & 16 \\
MIRI & $F 1000 W$ & 6 & 0.1 & 1 \\
\hline NIRCam & $F 115 W$ & 9 & 6 & 36 \\
NIRCam & $F 200 W$ & 9 & 7 & 42 \\
NIRCam & $F 480 M$ & 9 & 1 & 7 \\
MIRI & $F 1000 W$ & 9 & 0.06 & 0.5 \\
\hline
\end{tabular}

Notes. All values are computed for an initial stellar mass of $10^{8} M_{\odot}$ and for reference the CAN IMF and the MKDP IMF are considered. The $\mathrm{S} / \mathrm{N}$ values can be reached within a total integration time of $\approx 3 \mathrm{~h}$. Section 3.6 gives more details.

redshift. To quantify this we use the upcoming JWST as a benchmark. To cover the wavelength region computed here by PEGASE, the most suitable instrument is NIRCam in imaging mode, in total covering the region from 0.6 to $5 \mu \mathrm{m}$. To probe longer wavelengths, we also compute predictions for the MIRI instrument in imaging mode.

All predictions we make are for the UCDs with an initial stellar mass of $10^{8} M_{\odot}$; we use the CAN IMF as the standard and consider also the MKDP IMF. For the NIRCam instrument we use as a setup sub arrays FULL, readout DEEP8, groups 10, integration 1, and exposures 5. This results in a total exposure time of 10149 s. For the MIRI instrument the parameters are: subarrays FULL, readout FAST, groups 100, integration 1, exposures 36, resulting in a similar exposure time of $10090 \mathrm{~s}$. The predictions for the JWST telescope are summarized in Table 4. The general conclusion is that UCD progenitors are detectable using JWST photometry with $\mathrm{a} \approx 3 \mathrm{~h}$ exposure time with promising values of $\mathrm{S} / \mathrm{N}$, as already suggested for GCs progenitors by Renzini (2017). 


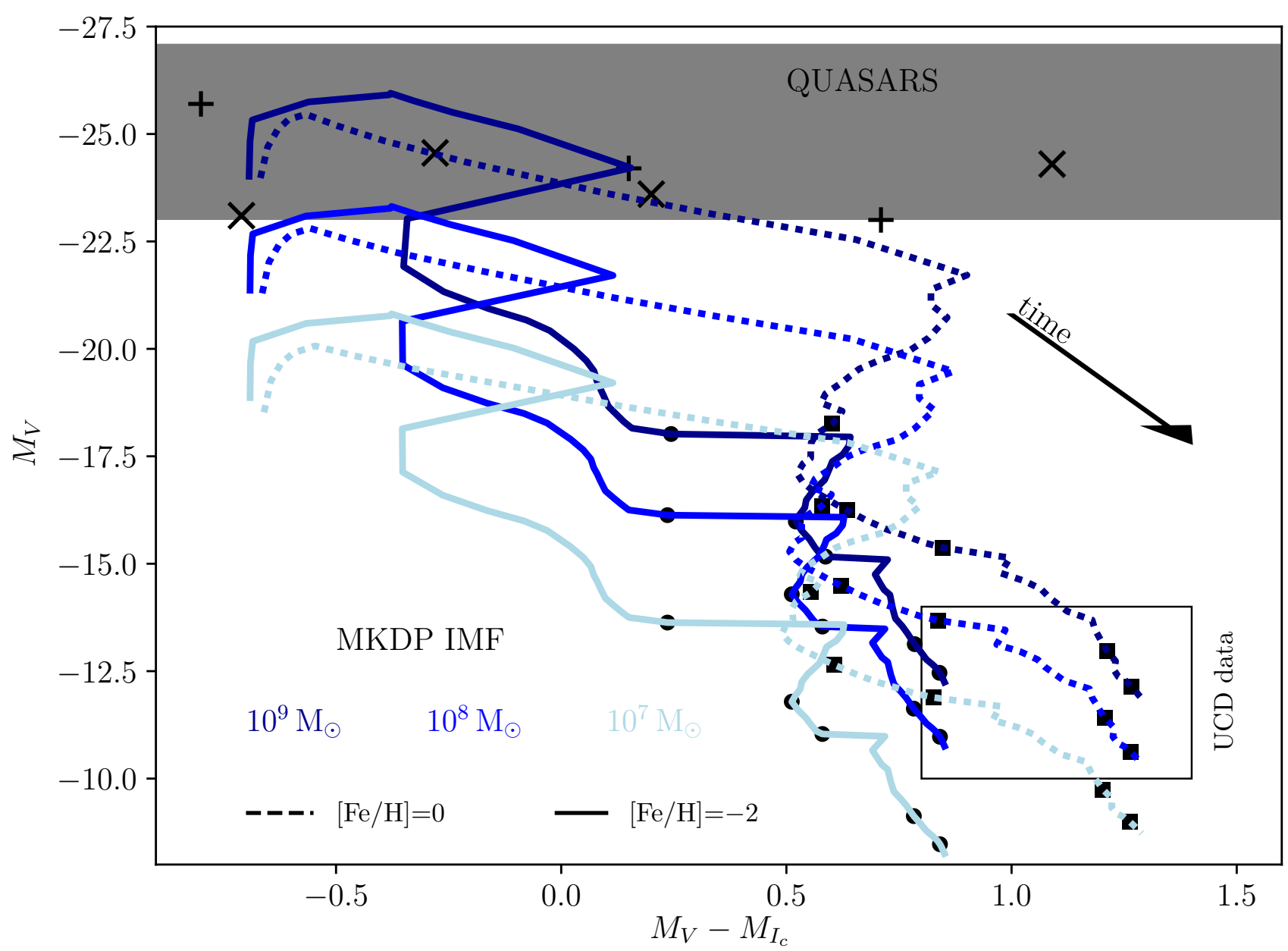

Fig. 9. Colour-magnitude diagram showing $M_{V}$ as a function of $V-I_{\mathrm{c}}$ (in the UCD rest frame). These latter are photometric filters directly computed by PEGASE. We consider $10^{7}, 10^{8}, 10^{9} M_{\odot}$ as initial stellar masses, and metallicity values $[\mathrm{Fe} / \mathrm{H}]=-2,0$. Here we show only the results for the MKDP IMF plotted together with the quasar data (black cross and plus markers, cross for radio quiet and plus for radio loud quasars) from Dunlop et al. (1993, 2003), Souchay et al. (2015). The data are compiled in Table 3. The CAN IMF, SAL IMF and vDC IMF are shown in Fig. 10. The arrow indicates the time evolution for the UCD models; the black filled circles and squares mark evolutionary time, from left to right: $100 \mathrm{Myr}, 500 \mathrm{Myr}, 1 \mathrm{Gyr}, 5 \mathrm{Gyr}, 10 \mathrm{Gyr}$ for $[\mathrm{Fe} / \mathrm{H}]=-2$ and 0 dex. The rectangular region indicates where the majority of observed UCDs are located (e.g. Evstigneeva et al. 2008). Since UCDs have a different metallicity, we do not plot individual data points.

\section{Discussion}

\subsection{Where to look}

The star formation rate density typically peaks near the centre of a galaxy. This is evident in interacting galaxies (Joseph \& Wright 1985; Norman 1987; Wright et al. 1988; Dabringhausen et al. 2012), while in self-regulated galaxies the distribution of starformation-rate density may be more complex as a result of converging gas flows, for example at the intersection points between a disc and bar. Generally though, central regions are the most active in star formation activity in star forming galaxies. That the most massive clusters form near the centres of galaxies where the SFR-density is highest is evident in various star-bursting galaxies (Ferrarese \& Merritt 2002; Dabringhausen et al. 2012), in our Galaxy (Stolte et al. 2014), and also in young-cluster surveys of individual galaxies (Pflamm-Altenburg et al. 2013). Simulations of star forming galaxies lead to the same result ( $\mathrm{Li}$ et al. 2017). Observationally it has been shown that the most massive clusters form preferentially in galaxies with the highest star formation rate (SFR) (Weidner et al. 2004; Randriamanakoto et al. 2013). We can therefore consider the following overall process: in the process of the formation of massive galaxies, star formation would have been spread throughout the merging proto-galactic gas clumps. The most massive clusters, the proto-UCDs, would be forming in the deepest potential wells of these, but would decouple from the hydrodynamics once they became stellar systems. As the proto-galaxies merge to form the massive central galaxies of galaxy clusters, many of such formed UCDs would end up on orbits about the central galaxy, possibly with the most massive UCDs within and near the centre of the galaxy.

We can therefore expect that the most massive UCDs, those that formed monolithically (i.e. according to formation scenario A), are to be found in the innermost regions of forming central-dominant galaxies at a high redshift. Such galaxies have been deduced to form on a timescale of and within less than a Gyr of the Big Bang with SFRs larger than a few $10^{3} M_{\odot} / \mathrm{yr}$ (Recchi et al. 2009). Under more benign conditions, that is when the system-wide SFR is smaller as in later interacting galaxies or the formation of less massive elliptical galaxies within a few Gyr of the Big Bang, UCDs may form also but are more likely to be the mergers of star-cluster complexes. Cases in point 

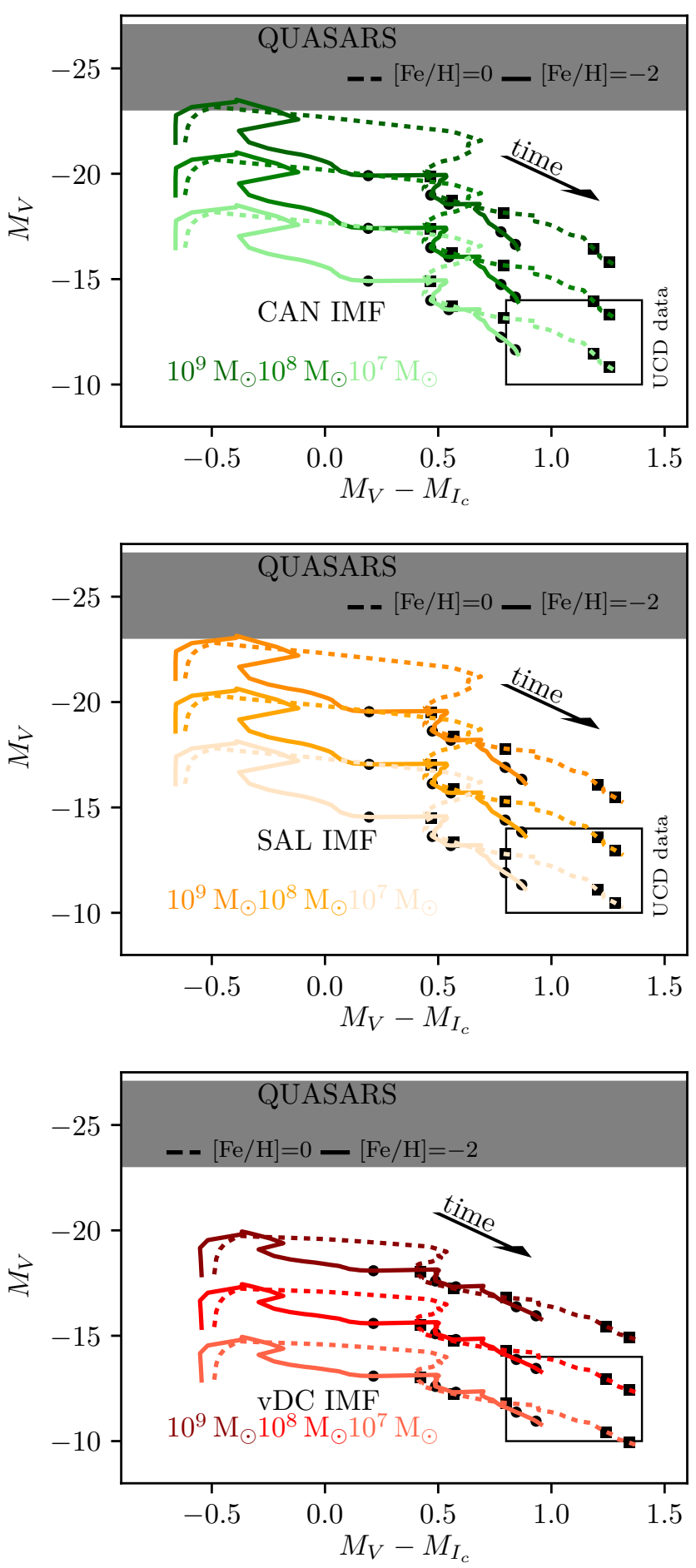

Fig. 10. As Fig. 9 but for the CAN IMF (MIRI, top panel), the SAL IMF (middle panel), and the vDC IMF (bottom panel).

are the Antennae galaxies, where such young complexes are evident (Kroupa 1998; Fellhauer \& Kroupa 2002b), and the Tadpole galaxy Kroupa (2015).

Therefore, the best place to search for very massive UCDs is in the inner regions of extreme star-bursts at very high redshift. Elliptical galaxies and bulges in formation may also host young massive UCDs. If it were possible to observationally remove the gas and dust obscuration, then such systems are likely to look like brilliantly lit Christmas trees.

\subsection{Which IMF is to be expected if the formation scenario $(A)$ or $(B)$ is realized?}

If monolithic collapse (scenario A) applies, then according to Marks et al. (2012), Dabringhausen et al. (2012) top-heavy MKDP IMFs are expected, which in turn lead to larger $M / L_{V}$ values at older ages (Figs. 4 and 5). At young ages, such objects can be as bright as quasars (Fig. 3).

In the case of the formation of UCDs from merged cluster complexes (scenario B), the IMF in each sub-cluster would be closer to the canonical IMF. The IMF of a whole object would thus be less top-heavy (e.g. Pflamm-Altenburg et al. 2009; Weidner et al. 2013; Fontanot et al. 2017; Yan et al. 2017), leading to smaller $M / L_{V}$ ratios at older ages (Figs. 4 and 5). They are significantly less luminous than monolithically-formed objects with MKDP IMFs (Fig. 3). Therefore the realization of both scenarios (A) and (B) in reality may lead to a spread of $M / L_{V}$ values for present-day UCDs, which may be comparable to the observed spread.

In the case of a bottom-heavy IMF, one might expect similar behaviour; that is, if a UCD is created by a monolithic star-burst the IMF may be more bottom-heavy than in the case of merged cluster complexes. Thus, for monolithically-formed objects, the $M / L_{V}$ ratios are large at all ages (Fig. 4), while they would be sub-luminous for their mass (Fig. 3).

All these different IMF cases can be distinguished best when the objects are younger than $100 \mathrm{Myr}$ as their luminosities and colours will be most different (Figs. 9, 10). Particularly useful observable diagnostics are provided by the colour-colour plots (Fig. A.8) and the slope of the SED (Fig. 8).

\subsection{The implications of observed $M / L_{V}$-ratios}

The elevated $M / L_{V}$ ratios observed for some UCDs can be caused by three, partially interconnected scenarios: (1) a variation of the IMF (top-heavy or bottom-heavy); (2) the presence of a super-massive black hole (SMBH); and (3) the presence of non-baryonic dark matter. Point (3) is directly connected to formation scenario (D), which is, as already mentioned, disfavoured for UCDs. As shown above, the variable IMF (scenario (1)) can explain the observed elevated $M / L_{V}$ values. The mass of a SMBH in scenario (2) required to explain the observed $M / L_{V}$ values, needs typically to be $10-15$ per cent of the present-day UCD mass (Mieske et al. 2013). The presence of SMBHs with such masses is indeed suggested or observationally confirmed at least for a few UCDs (e.g. Seth et al. 2014; Janz et al. 2015; Ahn et al. 2017).

To address which scenario (variation of IMF, (1), or presence of SMBH, (2)) is responsible for the elevated $M / L_{V}$ values in different formation scenarios is not straightforward. In the case of formation scenario (C), where UCDs are tidally stripped nuclei, there is an observational connection with the presence of SMBHs. Graham \& Spitler (2009) found that the existence of a $\mathrm{SMBH}$ in a galactic nuclear cluster is indeed frequently the case. However, since the exact formation mechanism of SMBHs is still discussed, it is not possible to constrain the IMF of UCDs formed by scenario (C). On the other hand, even though in formation scenarios (A) and (B), where UCDs are clusterlike objects and where the variable IMF is introduced to explain observed $M / L_{V}$ values, the existence of a SMBH cannot be excluded. The SMBH can potentially be formed as a merger of dark remnants (Giersz et al. 2015; Kroupa et al., in prep.). If a standard IMF is assumed, the mass of dark remnants (BHs and neutron stars) represents approximately only 2 per cent of the 
present-day mass of a system. For a top-heavy IMF this fraction can be significantly higher.

\section{Conclusions}

We investigated if observations with upcoming observatories, with an emphasis on predictions for the JWST, may be able to discern the formation and evolution of UCDs assuming that they are cluster-like objects which form by (A) single monolithic collapse or (and) (B) by the merging of cluster complexes. The primary area of interest is to find observable diagnostics that may allow us to assess how the stellar IMF varies with physical conditions. The extreme star-bursts, which massive GCs and UCDs must have been at a high redshift, may be excellent test beds for this goal. For this purpose we compute the time-dependent evolution of SEDs for different physical parameters and mainly for different IMFs. We test the top-heavy IMF, as parametrized by Marks et al. (2012), which predicts a top-heavy IMF for the case of scenario (A) and an IMF closer to the canonical IMF in the case of scenario (B). The bottom-heavy IMFs are implemented as a single power law function with a slope -2.3 (Salpeter) and -3 (van Dokkum \& Conroy 2010).

The main conclusions concerning the formation scenario for UCDs and the stellar IMF variability can be summarized in five main points:

- The retention fraction of stellar remnants is near to $100 \%$ for systems with birth masses larger than $10^{7} \mathrm{M}_{\odot}$.

- We show that if UCD progenitors younger than $\approx 100 \mathrm{Myr}$ are observed, their stellar IMF can be constrained and therefore also the formation scenario can be constrained by obtaining achievable measurements (e.g. absolute luminosity and supernova rate or an appropriate combination of colours and the value of $\beta_{\mathrm{UV}}$ ). UCD progenitors most likely located at redshifts 3-9 have not been observed yet, however, according to our predictions we should be able to detect them even with current telescopes as they would appear like point sources with high, quasar-like luminosities. Computed exposure times for chosen JWST, MIRI and NIRCam instruments are presented.

- We also discuss degeneracies, which start appearing at ages $>50 \mathrm{Myr}$ as massive stars and evolve into dark remnants, and we reveal which information and constraints we can obtain from present-day UCDs. That is, the object's luminosity with a top-heavy IMF starts to be comparable with a UCD of the same (or even smaller) initial mass but with a canonical or bottom-heavy IMF. Therefore, within observational uncertainties, these cases might be indistinguishable on colourmagnitude or colour-colour diagrams. Even $M / L_{V}$ becomes degenerate, however, for the majority of cases we should be able to separate a vDC IMF from the rest if the metallicity of the UCD is constrained reasonably well.

- If UCDs were formed with a top-heavy IMF ( $\alpha_{3}<2.3$ with the most extreme case considered $\alpha_{3}=0.6$ ), their progenitors are extreme and very different from Galactic star formation regions. The UCD progenitors with initial stellar masses of $\approx 10^{9} M_{\odot}$ would contain $\approx 10^{7} \mathrm{O}$ stars in a region spanning not more than a few pc. This drives a tremendous luminosity, very high SN II rates, and also poses the further question as to how the strong radiation field influences the state and evolution of other stars and thus the IMF especially at the low-mass end (e.g. Kroupa \& Bouvier 2003).

- Interestingly, we have found evidence that some of the observed quasars have photometric properties of very young
UCD models with top-heavy IMFs. This may suggest that some quasars at high redshift may actually be very massive UCDs with ages $<10 \mathrm{Myr}$. This needs further study though, for example by quantifications of SEDs. One method to help identify true UCDs with top-heavy IMFs would be to monitor their luminosities. Since core-collapse supernovae will be common in such systems, exploding at a rate of more than one per year, the luminosity of such a UCD ought to show increases by $\approx 0.1-10$ per cent (depending on star formation history, IMF, and initial stellar mass) over a timescale of a few months up to a few dozen times a year. Young UCDs should thus be time-variable.

- Groups of very young UCDs, if found at high redshift, may be indicating the assembly of the inner regions of galaxy clusters (see also Schulz et al. 2016): the assembly timescale is $10^{2} \mathrm{Myr}$, being of the order of the dynamical timescale. The seeds of the most massive galaxies in the centre of galaxy clusters probably had a very clustered formation of UCD-mass objects that created today's giant ellipticals and brightest cluster galaxies. Thus, during the assembly of the inner region of galaxy clusters, we would expect generations of quasar-like UCDs, each with a high luminosity and lifetime of about $10 \mathrm{Myr}$, forming such that the overall lifetime of the UCD-active epoch would be about $10^{2} \mathrm{Myr}$. This is comparable to the lifetime of quasars, adding to the similarity in photometric properties noted above.

- The majority of ultra-massive very young UCDs, which look comparable to quasars, are therefore likely to form in the central region of the star-bursts from which the present-day central dominant elliptical galaxies emerge. But such UCDs will not be observable today as they are likely to sink to the centres of the elliptical galaxies through dynamical friction Bekki (2010).

To gain more firm conclusions, individual cases of observed UCDs need to be considered taking all observational constraints into account. To disentangle degeneracies that arise mainly with age, new data reporting UCDs younger than $\approx 100 \mathrm{Myr}$ are needed. We would like to emphasize here that no such objects have been observationally confirmed yet.

Acknowledgements. We thank the referee and Holger Baumgardt for useful comments that helped to improve this manuscript. T.J. was supported by Charles University in Prague through grant SVV-260441 and through a stipend from the SPODYR group at the University of Bonn. T.J., P.K., and K.B. thank the DAAD (grant 57212729 "Galaxy formation with a variable stellar initial mass function") for funding exchange visits. We would like to acknowledge the use of Python (G. van Rossum, Python tutorial, Technical Report CS-R9526, Centrum voor Wiskunde en Informatica (CWI), Amsterdam, May 1995). Apart from standard Python libraries, we used pyPegase from Colin Jacobs. We also acknowledge discussions with Christopher Tout and many useful contributions seen during the ImBaSe 2017 conference in Garching.

\section{References}

Adams, F. C., \& Laughlin, G. 1996, ApJ, 468, 586

Ahn, C. P., Seth, A. C., den Brok, M., et al. 2017, ApJ, 839, 72

Anderson, J. P., Habergham, S. M., \& James, P. A. 2011, MNRAS, 416, 567

Balbinot, E., \& Gieles, M. 2017, MNRAS, submitted [arXiv: 1702 .02543]

Banerjee, S. 2017, MNRAS, 467, 524

Bastian, N., \& Strader, J. 2014, MNRAS, 443, 3594

Bastian, N., Covey, K. R., \& Meyer, M. R. 2010, ARA\&A, 48, 339

Baumgardt, H., \& Sollima, A. 2017, MNRAS, 472, 744

Bekki, K. 2010, MNRAS, 401, 2753

Bekki, K., Couch, W. J., \& Drinkwater, M. J. 2001, ApJ, 552, L105

Bekki, K., Couch, W. J., Drinkwater, M. J., \& Shioya, Y. 2003, MNRAS, 344 , 399

Bertelli Motta, C., Clark, P. C., Glover, S. C. O., Klessen, R. S., \& Pasquali, A. 2016, MNRAS, 462, 4171 
Brinkmann, N., Banerjee, S., Motwani, B., \& Kroupa, P. 2017, A\&A, 600, A49 Brodie, J. P., Romanowsky, A. J., Strader, J., \& Forbes, D. A. 2011, AJ, 142, 199 Brüns, R. C., Kroupa, P., Fellhauer, M., Metz, M., \& Assmann, P. 2011, A\&A, 529, A138

Chabrier, G., Hennebelle, P., \& Charlot, S. 2014, ApJ, 796, 75

Chiboucas, K., Tully, R. B., Marzke, R. O., et al. 2011, ApJ, 737, 86

Chilingarian, I. V., Cayatte, V., \& Bergond, G. 2008, MNRAS, 390, 906

Côté, P., Piatek, S., Ferrarese, L., et al. 2006, ApJS, 165, 57

Dabringhausen, J., Hilker, M., \& Kroupa, P. 2008, MNRAS, 386, 864

Dabringhausen, J., Kroupa, P., \& Baumgardt, H. 2009, MNRAS, 394, 1529

Dabringhausen, J., Fellhauer, M., \& Kroupa, P. 2010, MNRAS, 403, 1054

Dabringhausen, J., Kroupa, P., Pflamm-Altenburg, J., \& Mieske, S. 2012, ApJ, 747, 72

Dib, S., Kim, J., \& Shadmehri, M. 2007, MNRAS, 381, L40

Drinkwater, M. J., Jones, J. B., Gregg, M. D., \& Phillipps, S. 2000, PASA, 17, 227

Drinkwater, M. J., Gregg, M. D., Hilker, M., et al. 2003, Nature, 423, 519

Drinkwater, M. J., Gregg, M. D., Couch, W. J., et al. 2004, PASA, 21, 375

Dunlop, J. S., Taylor, G. L., Hughes, D. H., \& Robson, E. I. 1993, MNRAS, 264, 455

Dunlop, J. S., McLure, R. J., Kukula, M. J., et al. 2003, MNRAS, 340, 1095

Elmegreen, B. G. 2004, MNRAS, 354, 367

Evstigneeva, E. A., Drinkwater, M. J., Peng, C. Y., et al. 2008, AJ, 136, 461

Fellhauer, M., \& Kroupa, P. 2002a, Ap\&SS, 281, 355

Fellhauer, M., \& Kroupa, P. 2002b, MNRAS, 330, 642

Ferrarese, L., \& Merritt, D. 2002, Phys. World, 15N6, 41

Fioc, M., \& Rocca-Volmerange, B. 1997, A\&A, 326, 950

Fontanot, F., De Lucia, G., Hirschmann, M., et al. 2017, MNRAS, 464, 3812

Forbes, D. A., Lasky, P., Graham, A. W., \& Spitler, L. 2008, MNRAS, 389, 1924

Frank, M. J., Hilker, M., Mieske, S., et al. 2011, MNRAS, 414, L70

Gal-Yam, A. 2012, Science, 337, 927

Giersz, M., Leigh, N., Hypki, A., Lützgendorf, N., \& Askar, A. 2015, MNRAS, 454,3150

Glazebrook, K., Schreiber, C., Labbé, I., et al. 2017, Nature, 544, 71

Goerdt, T., Moore, B., Kazantzidis, S., et al. 2008, MNRAS, 385, 2136

Graham, A. W., \& Spitler, L. R. 2009, MNRAS, 397, 2148

Greggio, L., \& Renzini, A. 1983, A\&A, 118, 217

Hilker, M. 2009, in UCDs - A Mixed Bag of Objects, eds. T. Richtler, \& S. Larsen (Berlin, Heidelberg: Springer), 51

Hilker, M., Infante, L., Vieira, G., Kissler-Patig, M., \& Richtler, T. 1999, A\&AS, 134,75

Janz, J., Forbes, D. A., Norris, M. A., et al. 2015, MNRAS, 449, 1716

Joseph, R. D., \& Wright, G. S. 1985, MNRAS, 214, 87

Kroupa, P. 1998, MNRAS, 300, 200

Kroupa, P. 2001, MNRAS, 322, 231

Kroupa, P. 2015, Can. J. Phys., 93, 169

Kroupa, P., \& Bouvier, J. 2003, MNRAS, 346, 369

Kroupa, P., Weidner, C., Pflamm-Altenburg, J., et al. 2013, The Stellar and Sub-Stellar Initial Mass Function of Simple and Composite Populations (Dordrecht: Springer Science+Business Media), 115

Lamers, H. J. G. L. M., Gieles, M., Bastian, N., et al. 2005, A\&A, 441, 117

Larson, R. B. 1998, MNRAS, 301, 569

Leitherer, C., Schaerer, D., Goldader, J. D., et al. 1999, ApJS, 123, 3

Li, H., Gnedin, O. Y., Gnedin, N. Y., et al. 2017, ApJ, 834, 69

Liptai, D., Price, D. J., Wurster, J., \& Bate, M. R. 2017, MNRAS, 465, 105

Longmore, S. N. 2015, MNRAS, 448, L62

Lonsdale, C. J., Diamond, P. J., Thrall, H., Smith, H. E., \& Lonsdale, C. J. 2006, ApJ, 647, 185

Lyman, J. D., Bersier, D., James, P. A., et al. 2016, MNRAS, 457, 328

Maoz, D. 2008, MNRAS, 384, 267

Marks, M., \& Kroupa, P. 2012, A\&A, 543, A8
Marks, M., Kroupa, P., \& Oh, S. 2011, MNRAS, 417, 1684

Marks, M., Kroupa, P., Dabringhausen, J., \& Pawlowski, M. S. 2012, MNRAS, 422, 2246

Matteucci, F., \& Greggio, L. 1986, A\&A, 154, 279

Megeath, S. T., Gutermuth, R., Muzerolle, J., et al. 2016, AJ, 151, 5

Mieske, S., \& Kroupa, P. 2008, ApJ, 677, 276

Mieske, S., Hilker, M., \& Infante, L. 2002, A\&A, 383, 823

Mieske, S., Hilker, M., Jordán, A., Infante, L., \& Kissler-Patig, M. 2007, A\&A, 472, 111

Mieske, S., Dabringhausen, J., Kroupa, P., Hilker, M., \& Baumgardt, H. 2008, Astron. Nachr., 329, 964

Mieske, S., Hilker, M., \& Misgeld, I. 2012, A\&A, 537, A3

Mieske, S., Frank, M. J., Baumgardt, H., et al. 2013, A\&A, 558, A14

Misgeld, I., Mieske, S., Hilker, M., et al. 2011, A\&A, 531, A4

Mortlock, D. J., Warren, S. J., Venemans, B. P., et al. 2011, Nature, 474, 616

Murray, N. 2009, ApJ, 691, 946

Norman, C. A. 1987, in NASA Conference Publication, ed. C. J. Lonsdale Persson, 2466

Oh, K. S., Lin, D. N. C., \& Aarseth, S. J. 1995, ApJ, 442, 142

Papadopoulos, P. P. 2010, ApJ, 720, 226

Pejcha, O., \& Thompson, T. A. 2015, ApJ, 801, 90

Penny, S. J., Forbes, D. A., \& Conselice, C. J. 2012, MNRAS, 422, 885

Peuten, M., Zocchi, A., Gieles, M., Gualandris, A., \& Hénault-Brunet, V. 2016, MNRAS, 462, 2333

Pfeffer, J., \& Baumgardt, H. 2013, MNRAS, 433, 1997

Pfeffer, J., Griffen, B. F., Baumgardt, H., \& Hilker, M. 2014, MNRAS, 444, 3670

Pfeffer, J., Hilker, M., Baumgardt, H., \& Griffen, B. F. 2016, MNRAS, 458, 2492

Pflamm-Altenburg, J., Weidner, C., \& Kroupa, P. 2009, MNRAS, 395, 394

Pflamm-Altenburg, J., González-Lópezlira, R. A., \& Kroupa, P. 2013, MNRAS, 435, 2604

Planck Collaboration XIII. 2016, A\&A, 594, A13

Planck Collaboration Int. XLVII. 2016, A\&A, 596, A108

Price, J., Phillipps, S., Huxor, A., et al. 2009, MNRAS, 397, 1816

Randriamanakoto, Z., Escala, A., Väisänen, P., et al. 2013, ApJ, 775, L38

Recchi, S., Calura, F., \& Kroupa, P. 2009, A\&A, 499, 711

Renaud, F., Bournaud, F., \& Duc, P.-A. 2015, MNRAS, 446, 2038

Renzini, A. 2017, MNRAS, 469, L63

Renzini, A., D’Antona, F., Cassisi, S., et al. 2015, MNRAS, 454, 4197

Romano, D., Matteucci, F., Zhang, Z. Y., Papadopoulos, P. P., \& Ivison, R. J. 2017, MNRAS, 470, 401

Schulz, C., Pflamm-Altenburg, J., \& Kroupa, P. 2015, A\&A, 582, A93

Schulz, C., Hilker, M., Kroupa, P., \& Pflamm-Altenburg, J. 2016, A\&A, 594, A119

Seth, A. C., van den Bosch, R., Mieske, S., et al. 2014, Nature, 513, 398

Smith, R. J., \& Lucey, J. R. 2013, MNRAS, 434, 1964

Souchay, J., Andrei, A. H., Barache, C., et al. 2015, A\&A, 583, A75

Stanway, E. R., Eldridge, J. J., \& Becker, G. D. 2016, MNRAS, 456, 485

Stolte, A., Hußmann, B., Morris, M. R., et al. 2014, ApJ, 789, 115

Terlevich, R. J., \& Boyle, B. J. 1993, MNRAS, 262, 491

Thielemann, F.-K., Nomoto, K., \& Yokoi, K. 1986, A\&A, 158, 17

Thies, I., Pflamm-Altenburg, J., Kroupa, P., \& Marks, M. 2015, ApJ, 800, 72

Thomas, P. A., Drinkwater, M. J., \& Evstigneeva, E. 2008, MNRAS, 389, 102

van Dokkum, P. G., \& Conroy, C. 2010, Nature, 468, 940

Vanzella, E., Calura, F., Meneghetti, M., et al. 2017, MNRAS, 467, 4304

Weidner, C., Kroupa, P., \& Larsen, S. S. 2004, MNRAS, 350, 1503

Weidner, C., Kroupa, P., Pflamm-Altenburg, J., \& Vazdekis, A. 2013, MNRAS, 436, 3309

Wright, G. S., Joseph, R. D., Robertson, N. A., James, P. A., \& Meikle, W. P. S. 1988, MNRAS, 233, 1

Yan, Z., Jerabkova, T., \& Kroupa, P. 2017, A\&A, 607, A126

Zhang, Y., \& Bell, E. F. 2017, ApJ, 835, L2 


\section{Appendix A: Additional figures and procedures}

To make the text of the main paper more continuous, we present a part of the figures in this Appendix. At first we would like to present here redshift as a function of time from the Big-Bang. Even though this plot is elementary, it is not straightforward to find it in other literature in this format. We marked the redshifttime points that are relevant for this study.

In addition, since the PEGASE SED's minimal wavelength is $\approx 1000 \AA$, and because at higher redshifts $(z \gtrsim 6)$ this region contributes to the standard optical filters, we extrapolate the computed SEDs at smaller wavelengths using a black-body approximation. To avoid adding an additional uncertainty to our results, we do not use the extrapolations to make any predictions or conclusions, but strictly as a demonstration of an approximate trend.

As a consistency check and also as a basic estimation of the difference between different stellar population codes, we computed the same set of SEDs with the StarBurst99-code (Leitherer et al. 1999) online library. The results are plotted in Fig. A. 2 showing agreement in the general characteristic of the SED and in the time evolution. The maximum differences in the SEDs, estimated only in the region which is computed by PEGASE without extrapolating, reach a factor of a few and are expected since different stellar evolutionary tracks are used in both codes. These differences represent the minimal uncertainty that needs to be considered if our results are compared with observations.

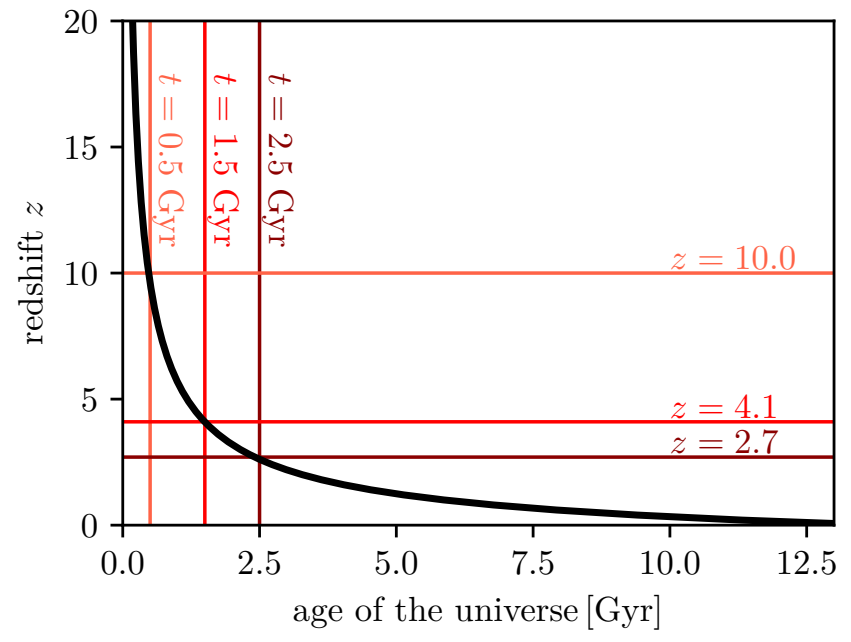

Fig. A.1. Redshift $z$ as a function of time from the Big-Bang (age of the Universe) adopting for this study the standard $\Lambda$ CDM cosmology with Planck parameters (see Sect. 2.1).

The time evolution of SEDs for the MKDP IMF is plotted in Fig. A.3 in comparison to the CAN IMF. The same time evolution for the vDC and SAL IMFs is plotted in Figs. A.5 and A.6. The wavelength shift and fainting proportional to the inverse square of luminosity distance with redshift is shown in Fig. A.4. 


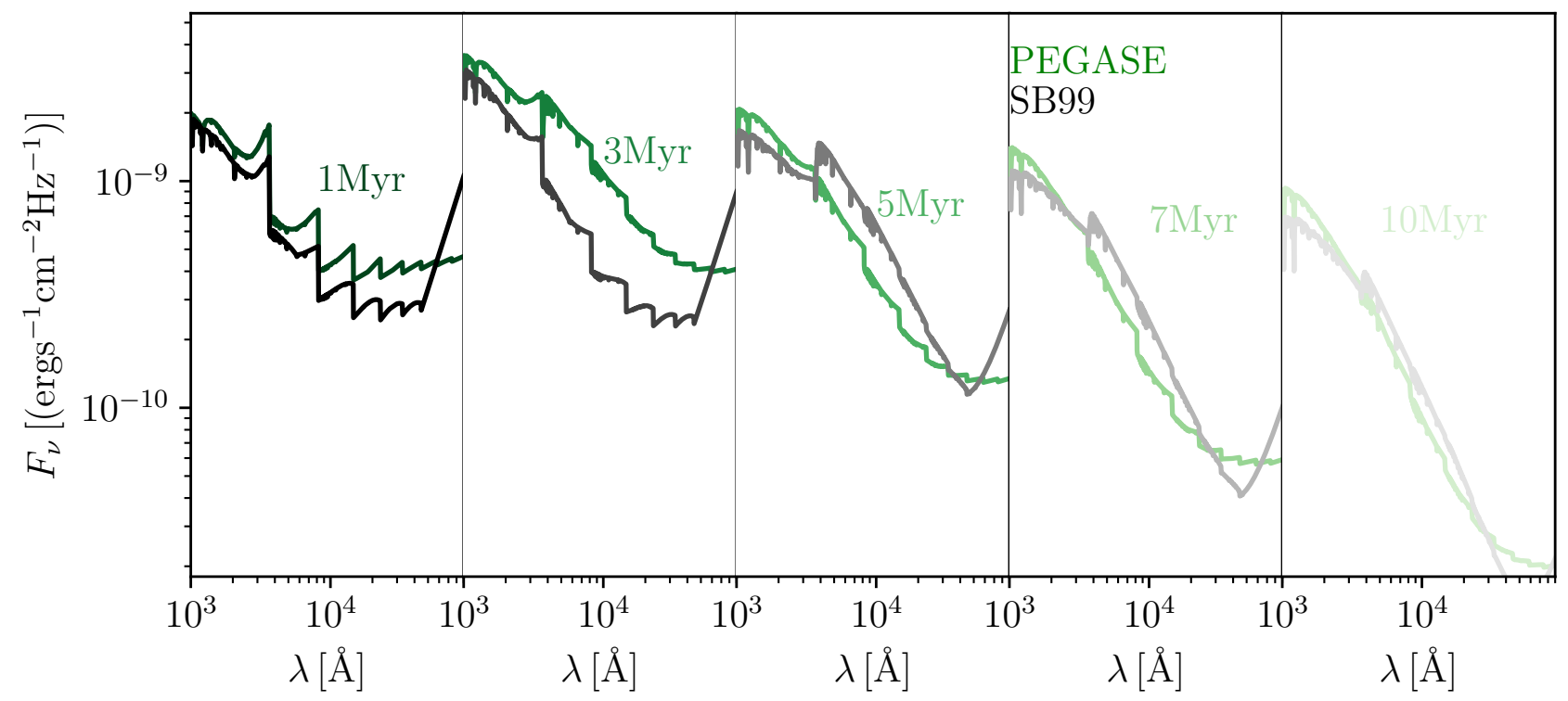

Fig. A.2. Time evolution of SEDs for a $10^{9} M_{\odot}$ system normalized to the distance of $10 \mathrm{pc}$ for the SAL IMF slope $\alpha=2.35$ within the mass range $(1,100) M_{\odot}$ and metallicity $Z=0.001$. We compare computed PEGASE models and downloaded SB99 models with the same parameters. The SEDs are normalized to the distance of $10 \mathrm{pc}$. The stellar mass range used here has been adopted only for this comparison for computational ease.

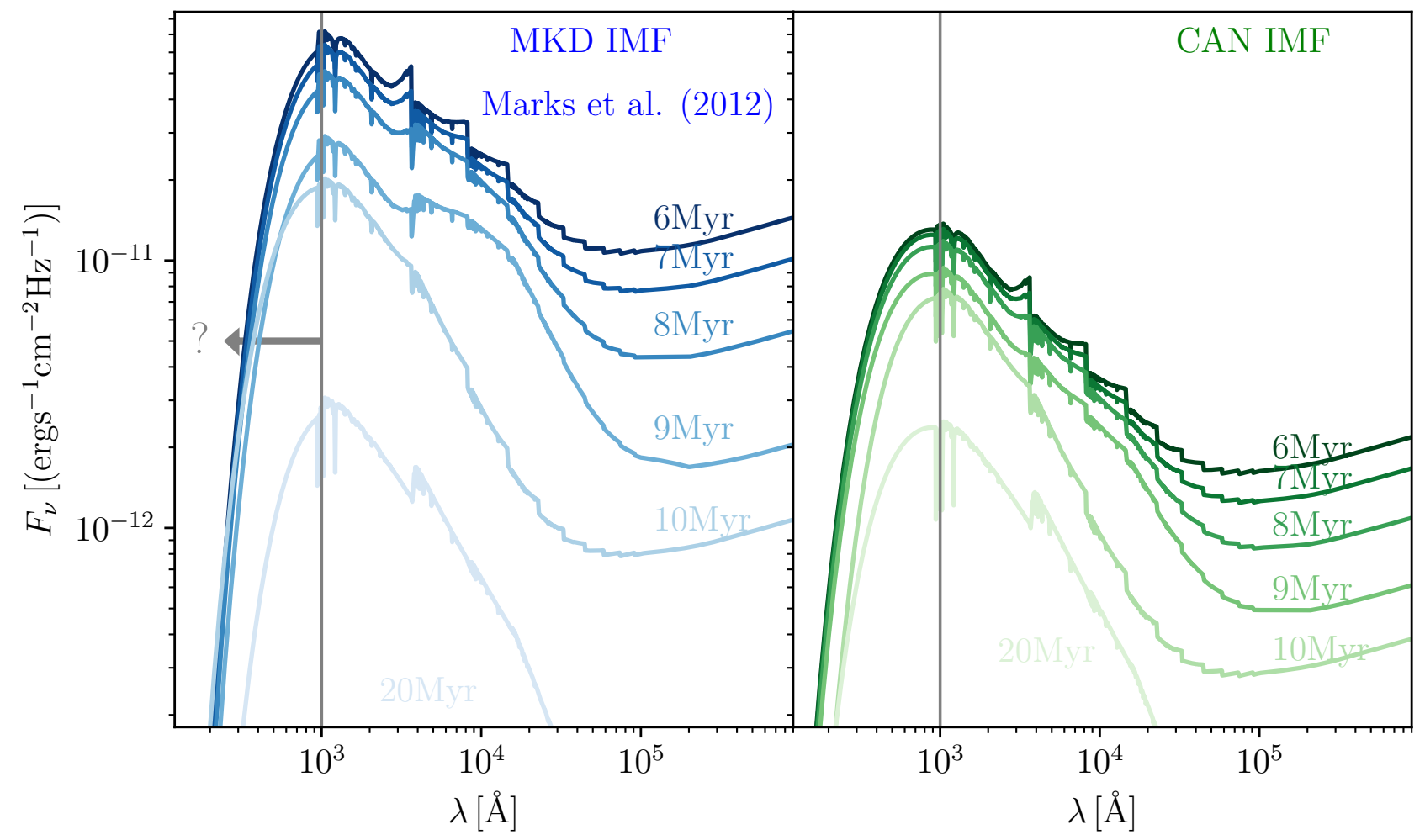

Fig. A.3. PEGASE time evolution of SEDs for $[\mathrm{Fe} / \mathrm{H}]=-2$ and a representative initial stellar mass of $10^{7} M_{\odot}$ as if it is at distance of $10 \mathrm{pc}$. Since we assume that the star formation lasts for $5 \mathrm{Myr}$, we start with the SED at $6 \mathrm{Myr}$ to avoid overlapping of lines. Left panel: the evolution for the MKDP IMF. Right panel: the evolution for the CAN IMF. The region below a wavelength of $\approx 1000 \AA$ (marked by arrow and question mark) is the black-body approximation. The PEGASE code does not predict values in this region. More details are given in the text. 
T. Jeřábková et al.: The formation of UCDs: Testing for a variable stellar IMF

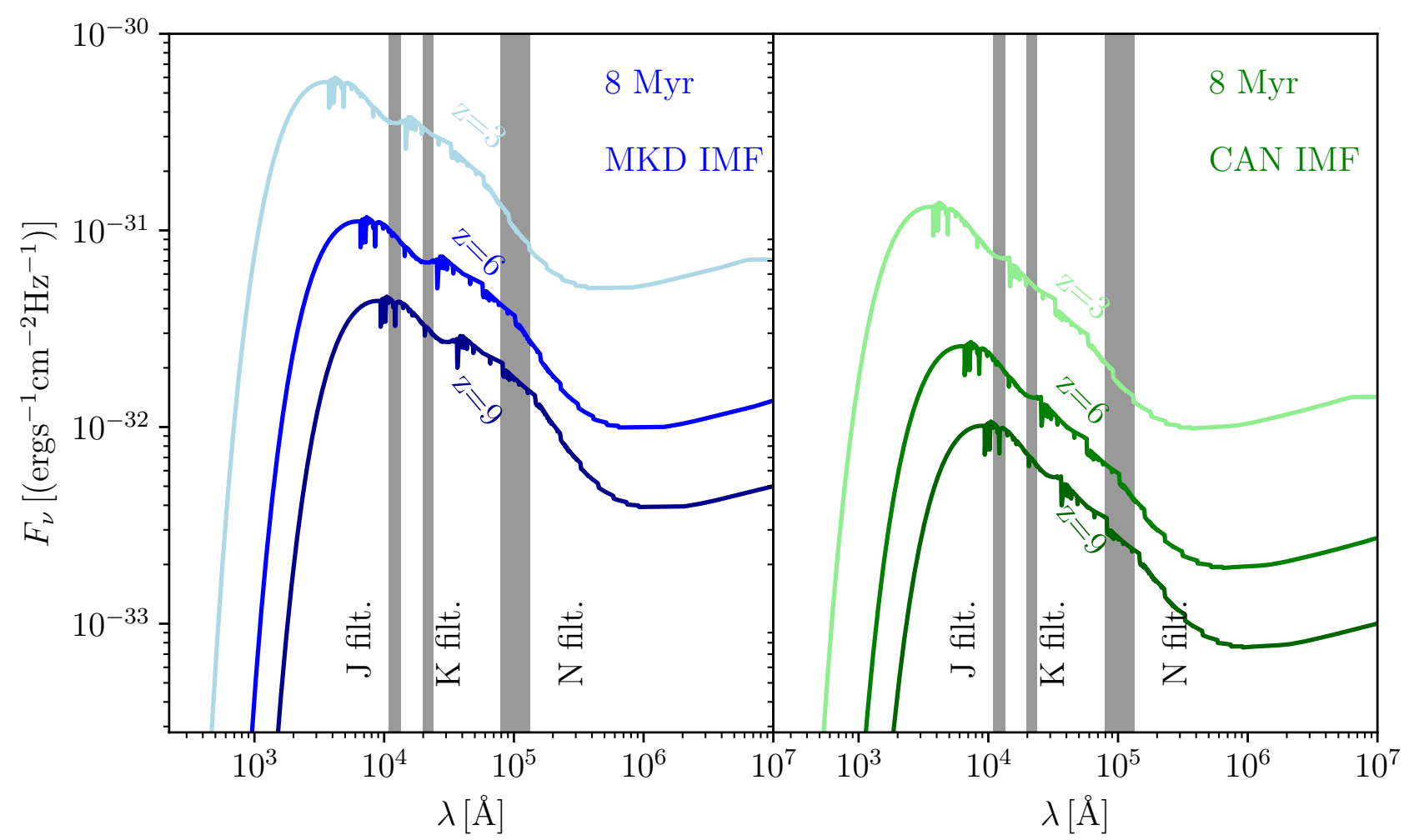

Fig. A.4. SEDs of 8-Myr-old UCDs at different redshifts. For this purpose, we arbitrarily chose the SED of an 8-Myr-old stellar population with metallicity $[\mathrm{Fe} / \mathrm{H}]=-2$ and corrected the spectrum for the wavelength shift and luminosity fainting with luminosity distance for redshifts 3, 6, and 9. Left panel: SEDs of the MKDP IMF. Right panel: SEDs of the CAN IMF. The photometric filters, here approximated by rectangular profiles as shaded vertical regions, are shown. The $J, K$, and $N$ filters are used in the colour analysis of the data.

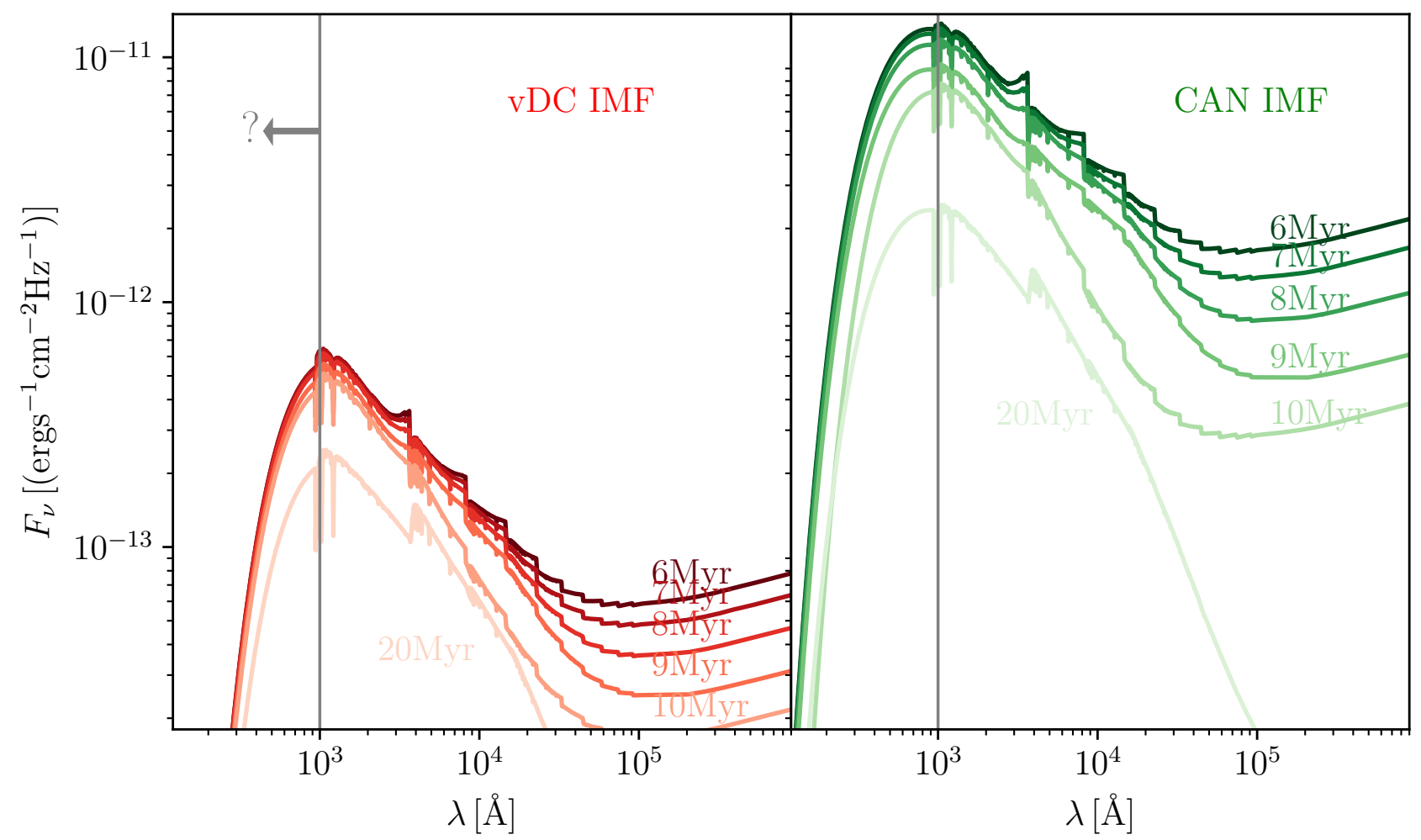

Fig. A.5. As Fig. A.3 but for the vDC IMF (left panel), with the CAN IMF (right panel, identical to Fig. A.3) shown here as a benchmark. 


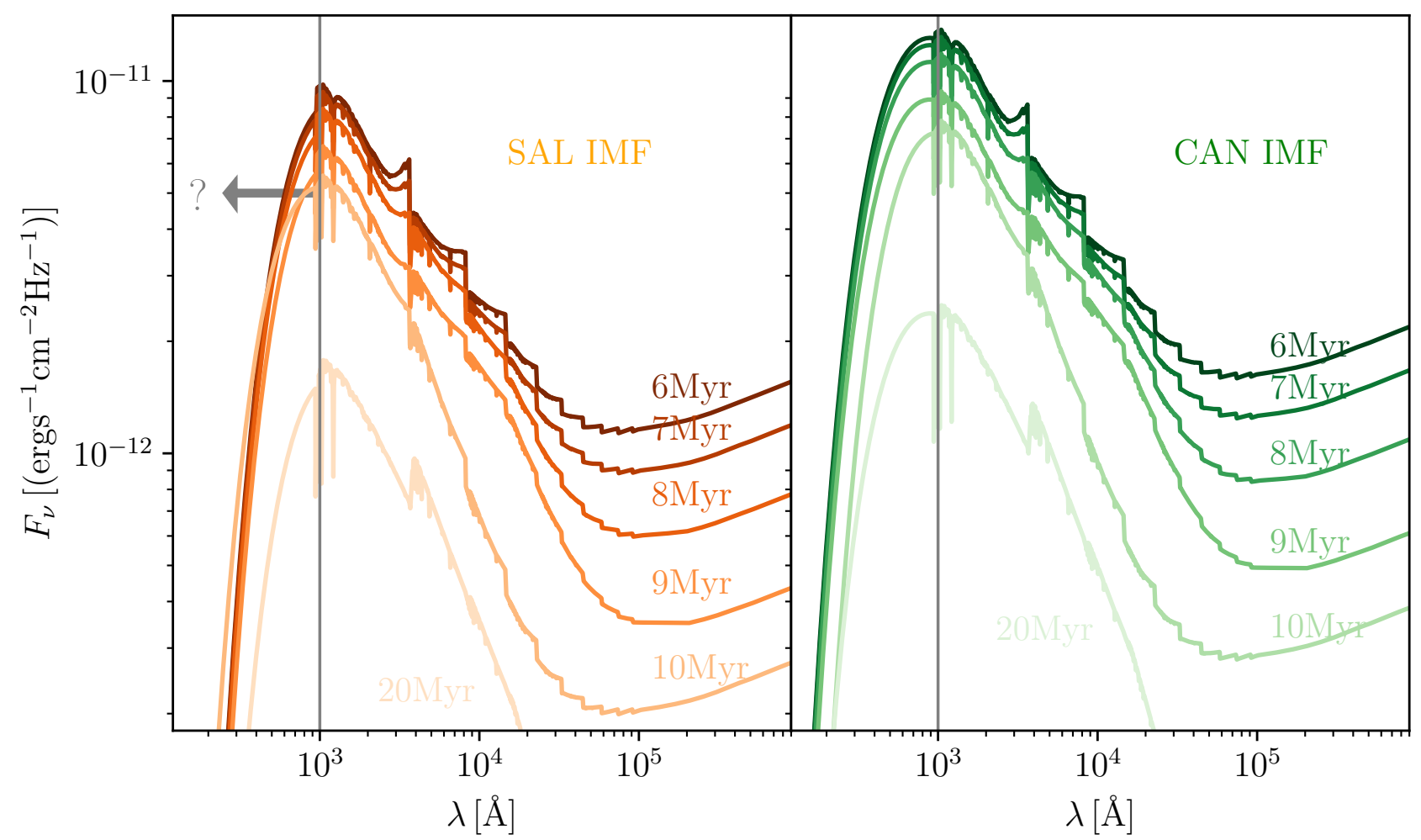

Fig. A.6. As Fig. A.3 but for the SAL IMF (left panel), with the CAN IMF (right panel, identical to Fig. A.3) shown here as a benchmark.

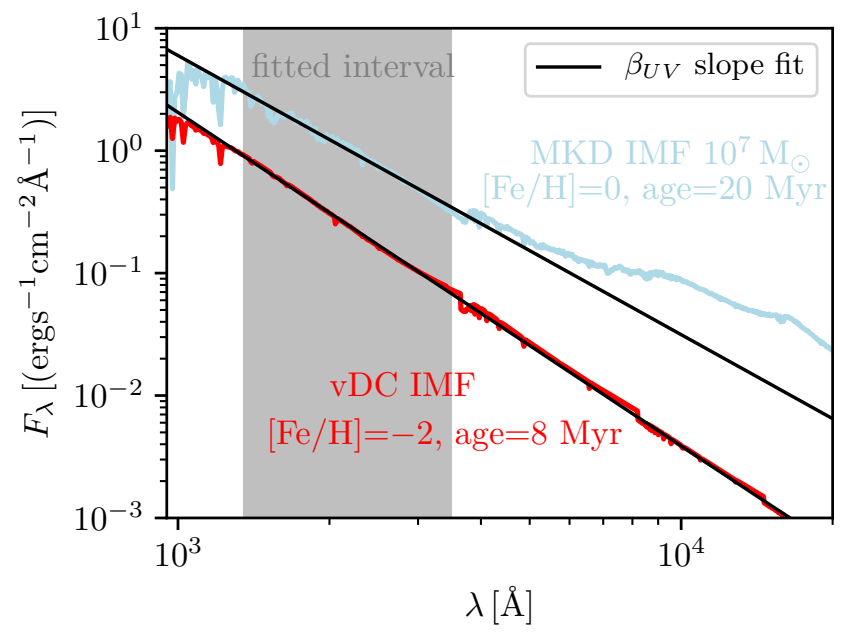

Fig. A.7. Fit to a SED in the grey shaded region shows the slope $\beta_{\mathrm{UV}}$ for two different UCDs with different age, the same mass $10^{7} M_{\odot}$, and different metallicity. We can see that in the grey region the spectra have a smooth shape and therefore it is possible to fit this part by a linear function to obtain a good estimate of $\beta_{\mathrm{UV}}$. Section 3.4 gives more details. 
T. Jeřábková et al.: The formation of UCDs: Testing for a variable stellar IMF
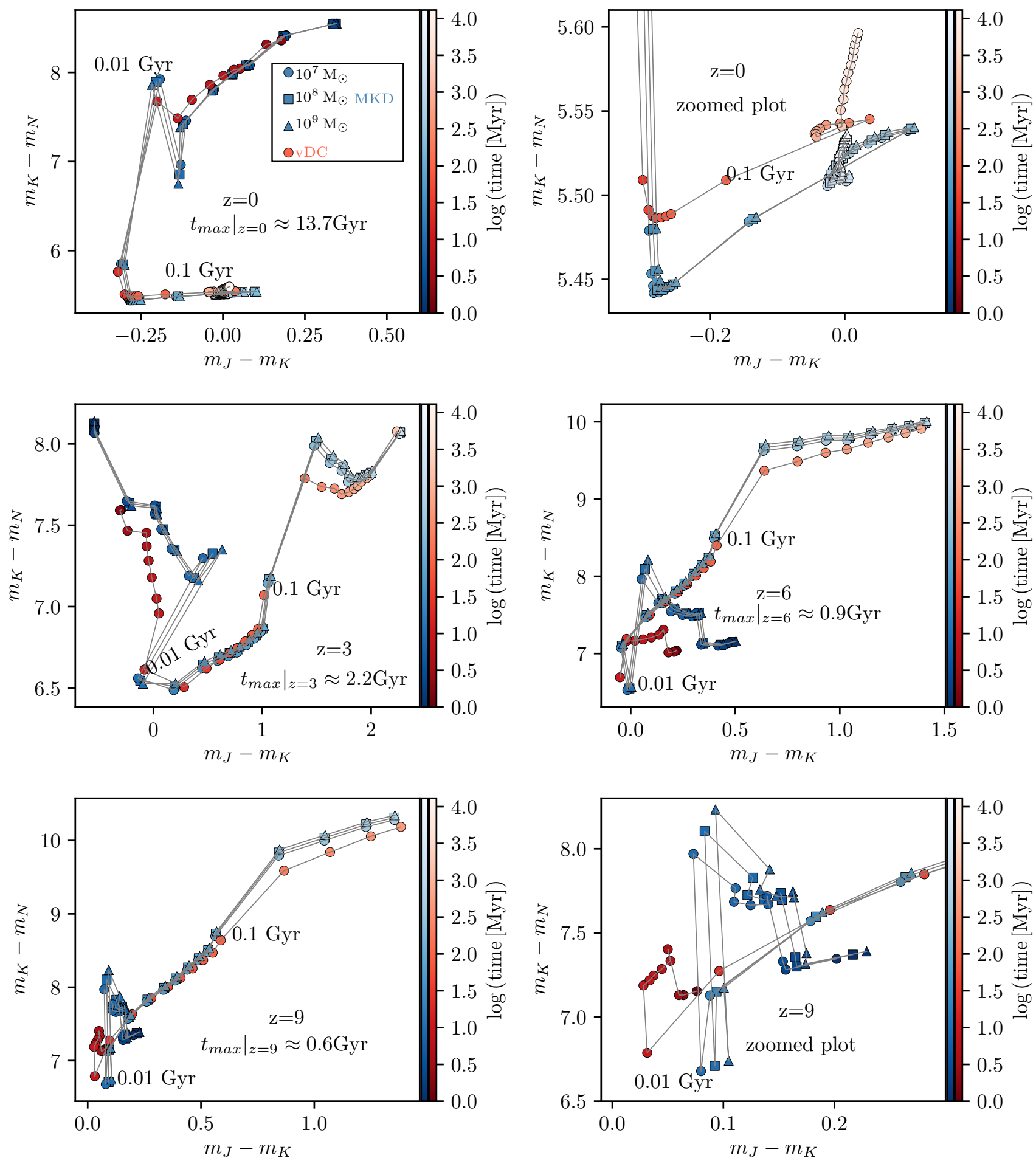

Fig. A.8. Colour-colour diagram made for standard filters $J, K$, and $N$ approximated here by rectangular boxes in Fig. A.4 showing the comparison of the vDC IMF and the MKDP IMF for different initial UCD masses, $M_{\mathrm{UCD}}$. Since according to the $\Lambda$ CDM cosmological model the upper limit to the age of the universe is $t_{\max } \approx 13.7 \mathrm{Gyr}$, we plot only ages consistent with this constraint on a corresponding redshift (here values are plotted at a redshift of $0,3,6$, and 9). The age evolution is shown by a colour scale that is the same for all plots. 